\title{
Communication \\ Development of Anthraquinone Analogues as Phosphoglycerate Mutase 1 Inhibitors
}

\author{
Ke Huang, Lulu Jiang, Huiti Li, Deyong Ye * and Lu Zhou* \\ Department of Medicinal Chemistry, School of Pharmacy, Fudan University, No. 826, Zhangheng Rd., \\ Shanghai 201203, China; kehuang13@fudan.edu.cn (K.H.); 18111030012@fudan.edu.cn (L.J.); \\ 17211030005@fudan.edu.cn (H.L.) \\ * Correspondence: dyye@shmu.edu.cn (D.Y.); zhoulu@fudan.edu.cn (L.Z.); Tel.: +86-21-5198-0117 (D.Y.); \\ +86-21-5198-0125 (L.Z.)
}

Received: 18 December 2018; Accepted: 28 January 2019; Published: 27 February 2019

check for updates

\begin{abstract}
Phosphoglycerate mutase 1 (PGAM1) coordinates glycolysis and biosynthesis to promote cancer cell proliferation, and is believed to be a promising target for cancer therapy. Herein, based on the anthraquinone scaffold, we synthesized 31 anthraquinone derivatives and investigated the structure-activity relationship (SAR). The 3-substitient of sulfonamide on the anthraquinone scaffold was essential for maintaining potency and the modifications of the hydroxyl of alizarin would cause a sharp decrease in potency. In the meantime, we determined the co-crystal structure of PGAM1 and one of the anthraquinone inhibitors $9 \mathrm{i}$ with $\mathrm{IC}_{50}$ value of $0.27 \mu \mathrm{M}$. The co-crystal structure revealed that F22, K100 and R116 of PGAM1 were critical residues for the binding of inhibitors which further validated the SAR. Consistent with the crystal structure, a competitive assay illustrated that compound $\mathbf{9} \mathbf{i}$ was a noncompetitive inhibitor. In addition, compound $\mathbf{9 i}$ effectively restrained different lung cancer cells proliferation in vitro. Taken together, this work provides reliable guide for future development of PGAM1 inhibitors and compound 9i may act as a new leading compound for further optimization.
\end{abstract}

Keywords: phosphoglycerate mutase 1; anthraquinone inhibitors; co-crystal structure; cancer treatment

\section{Introduction}

In 1924, Warburg discovered that cancer cells tended to metabolize glucose through aerobic glycolysis rather than oxidative phosphorylation, even if there was sufficient oxygen [1,2]. This character of cancer cells was distinct from normal differentiated cells and the phenomenon was thereafter named the "Warburg effect". However, the reason why cancer cells adopt such an altered metabolism remained unclear. In the past decades, reprogramming energy metabolism was considered as an emerging hallmark of cancer cells [3] and the study of cancer metabolism has drawn great interest [4-8]. During aerobic glycolysis of cancer cells, adenosine 5'-triphosphate (ATP) is produced inefficiently in this metabolic pattern, whereas building blocks such as nucleotides, and amino acids are generated in large amounts, satisfying the demands of anabolic biosynthesis which are essential for rapid cell proliferation [9-12]. Nowadays, various enzymes playing critical roles in cancer metabolism have been reported, including isocitrate dehydrogenase [13-15]. Inhibitors targeting these enzymes have been approved by Food and Drug Administration [14,15] which further demonstrated the potential of modulating cancer metabolism for cancer therapy.

Phosphoglycerate mutase 1 (PGAM1) is a key enzyme in the 8th step of glycolysis pathway converting 3-phosphoglycerate (3PG) to 2-phosphoglycerate (2PG) [16]. Previously we found that PGAM1 could balance the intracellular concentrations of its substrate 3PG and its product 2PG to control tumor growth [17]. In detail, 3PG inhibits 6-phosphogluconate dehydrogenase in 
pentose phosphate pathway (PPP) and 2PG activates 3-phosphoglycerate dehydrogenase in serine synthesis [17]. When PGAM1 is inhibited by a small molecule or shRNA, intracellular level of 3PG increased and 2PG decreased which led to the blockade of PPP, serine synthesis, glycolysis and subsequently impaired tumor growth. Since PGAM1 regulates both glycolysis and biosynthesis, developing inhibitors targeting PGAM1 is considered as a way of dual inhibition of catabolism and anabolism for cancer therapy [18]. Furthermore, PGAM1 is upregulated in many kinds of tumors and the expression of PGAM1 is correlated with cancer patients' prognosis [19-21]. Recently, more functions of PGAM1 in cancer metabolism were described. Posttranslational modulations of PGAM1 such as Y26 phosphorylation [22] and deacetylation of K100 [23] would promote cancer cell proliferation and tumor growth. Besides, it is essential for homologous recombination repair by regulating the deoxyribonucleotide triphosphate nucleoside (dNTP) pool [24] and it facilitates cancer cell migration independent of its metabolic function [25]. Taken together, PGAM1 is a promising target for cancer treatment.

However, up to now, only four representative PGAM1 inhibitors were reported (Figure 1). The first PGAM1 inhibitor 1 (MJE3) was discovered by the Cravatt group in 2005 [26]. It was identified as a covalent inhibitor labeling the K100 of PGAM1 by spiroepoxides [27]. Another PGAM1 inhibitor described these days was 2((-)-epigallocatechin-3-gallate (EGCG)), a natural product from green tea [28]. It demonstrated strong inhibition activity towards PGAM1 [28] but it was reported to display extensive potency on multiple targets [29-31]. We discovered the other two inhibitors, one of which was anthraquinone derivative 3 (PGMI-004A) [17] and the other was the xanthone derivative 4 (12r) [32]. Both of them showed moderate inhibition activity on PGAM1 and cancer cell proliferation.

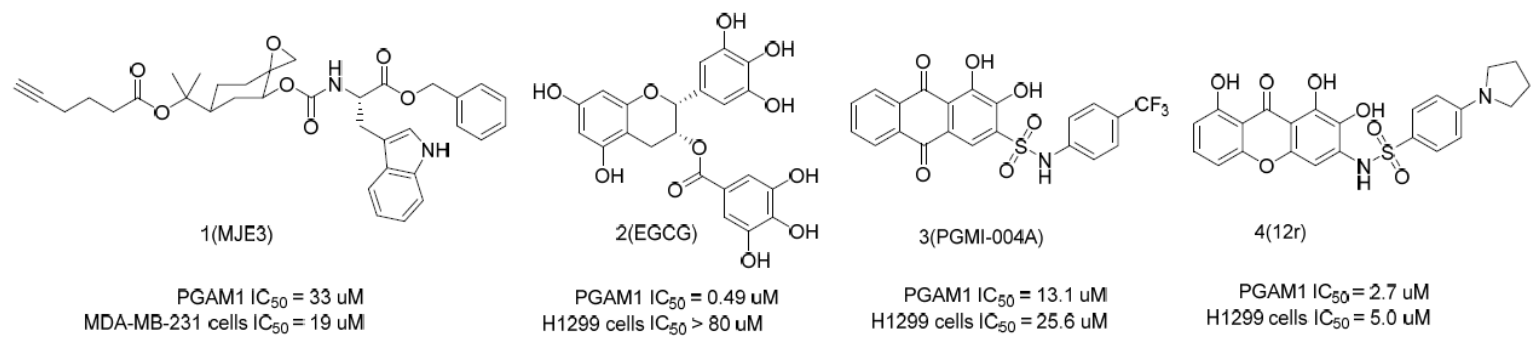

Figure 1. Reported phosphoglycerate mutase 1 (PGAM1) inhibitors.

Since only a limited amount of anthraquinone inhibitors were reported, the structure-activity relationship (SAR) of anthraquinone derivatives and PGAM1 was not well studied yet. Besides, up to these days, the molecular interactions of PGAM1 and anthraquinone inhibitor were not observed in crystal structure. However, anthraquinones exhibit a variety of pharmacological activities including anticancer [33-35], antifungal [36], and antibacterial properties [37] and so on. What's more, there are many clinically approved anthraquinone drugs such as doxorubicin and mitoxantrone with strong potency in suppressing cancer cell proliferation [38]. Here we designed and synthesized a series of new anthraquinone compounds and evaluated their biological activity on PGAM1 and cancer cell proliferation. In addition, we solved the co-crystal structure of PGAM1 and compound 9i which interpreted the molecular mechanism of compound 9i interacting with PGAM1 and further confirmed the SAR we investigated. Combined with the crystal structure, additional competitive assay demonstrated the noncompetitive binding of compound 9i with the 3PG substrate.

\section{Results and Discussion}

\subsection{Chemistry}

Four classes of anthraquinone derivatives were synthesized, with 1,2-substituted, 3-substituted, 2,4-substituted, and 2,3-substituted anthraquinone core scaffolds (Figure 2). 

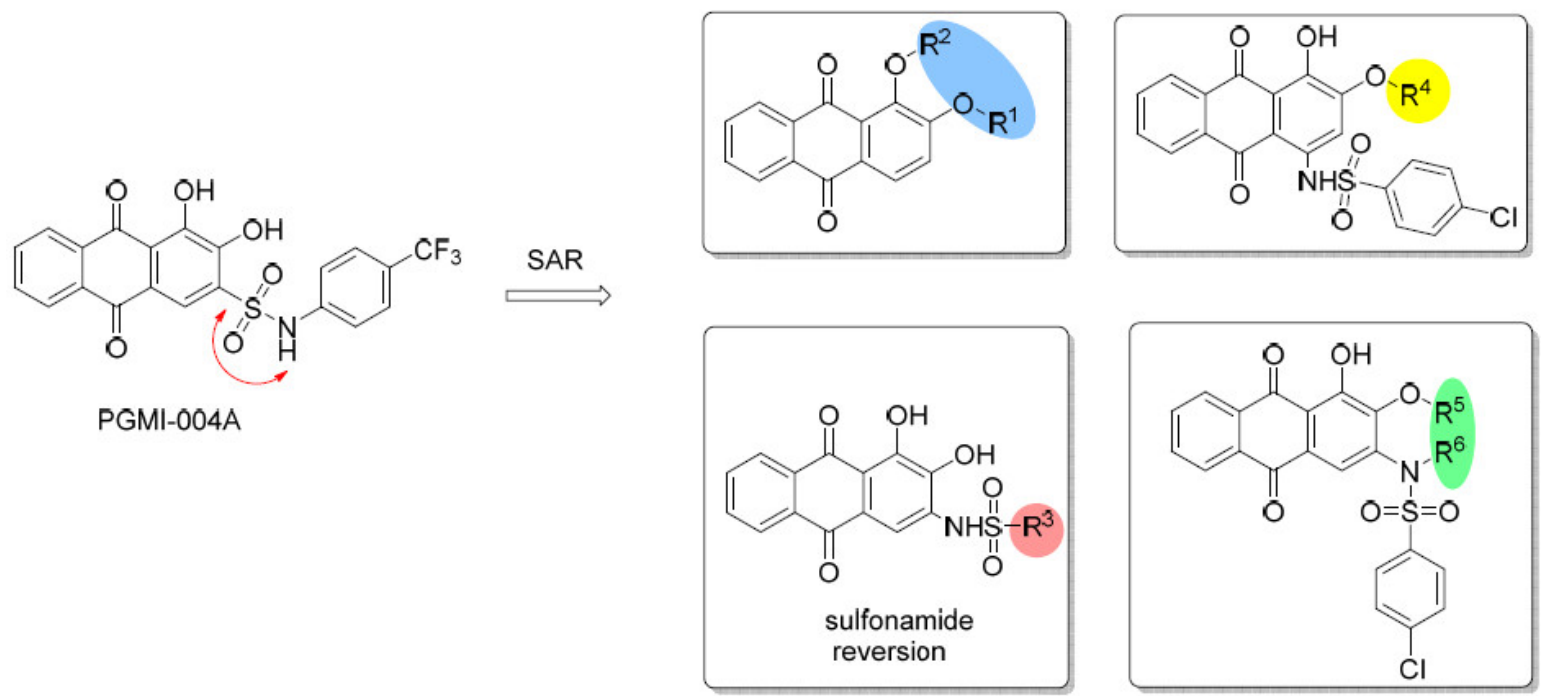

Figure 2. The optimization strategy and SAR exploration of the anthraquinone derivatives against PGAM1.

As shown in Scheme 1, starting from commercially available alizarin, compounds 6a-e were obtained by substitution with ethyl bromoacetate, hydrolysis of the ester, and amidation.<smiles>O=C1c2ccccc2C(=O)c2c1ccc(O)c2O</smiles><smiles>[R]Oc1ccc2c(c1O[R])C(=O)c1ccccc1C2=O</smiles>

b $\left(\begin{array}{l}\text { 6a: } \mathrm{R}^{1}=\mathrm{CH}_{2} \mathrm{COOEt}, \mathrm{R}^{2}=\mathrm{CH}_{2} \mathrm{COOEt} \\ \text { 6b: } \mathrm{R}^{1}=\mathrm{CH}_{2} \mathrm{COOH}, \mathrm{R}^{2}=\mathrm{CH}_{2} \mathrm{COOH}\end{array}\right.$

b $\left(6 \mathrm{c}: \mathrm{R}^{1}=\mathrm{CH}_{2} \mathrm{COOEt}, \mathrm{R}^{2}=\mathrm{H}\right.$

$6 \mathrm{~d}: \mathrm{R}^{1}=\mathrm{CH}_{2} \mathrm{COOH}, \mathrm{R}^{2}=\mathrm{H}$

c $6 \mathrm{e}: \mathrm{R}^{1}=\mathrm{CH}_{2} \mathrm{CONMe}_{2}, \mathrm{R}^{2}=\mathrm{H}$

Scheme 1. Synthesis of compounds 6a-e. Reagents and conditions: (a) $\mathrm{BrCH}_{2} \mathrm{COOEt}, \mathrm{K}_{2} \mathrm{CO}_{3}, \mathrm{DMF}$, $80{ }^{\circ} \mathrm{C}$; (b) $\mathrm{LiOH}, \mathrm{MeOH}, \mathrm{H}_{2} \mathrm{O}, 30^{\circ} \mathrm{C}$; (c) $\mathrm{NHMe}_{2} \cdot \mathrm{HCl}, \mathrm{EDCI}, \mathrm{HOBt}$, DIPEA, DMF.

In Scheme 2, firstly, alizarin was nitrated by $\mathrm{HNO}_{3}$ and then reduced by $\mathrm{Sn}$ and $\mathrm{HCl}$ to afford the key amine intermediate 8 , which was reacted with diverse sulfonyl chlorides to give the target compounds $9 \mathbf{a}-\mathbf{q}$.

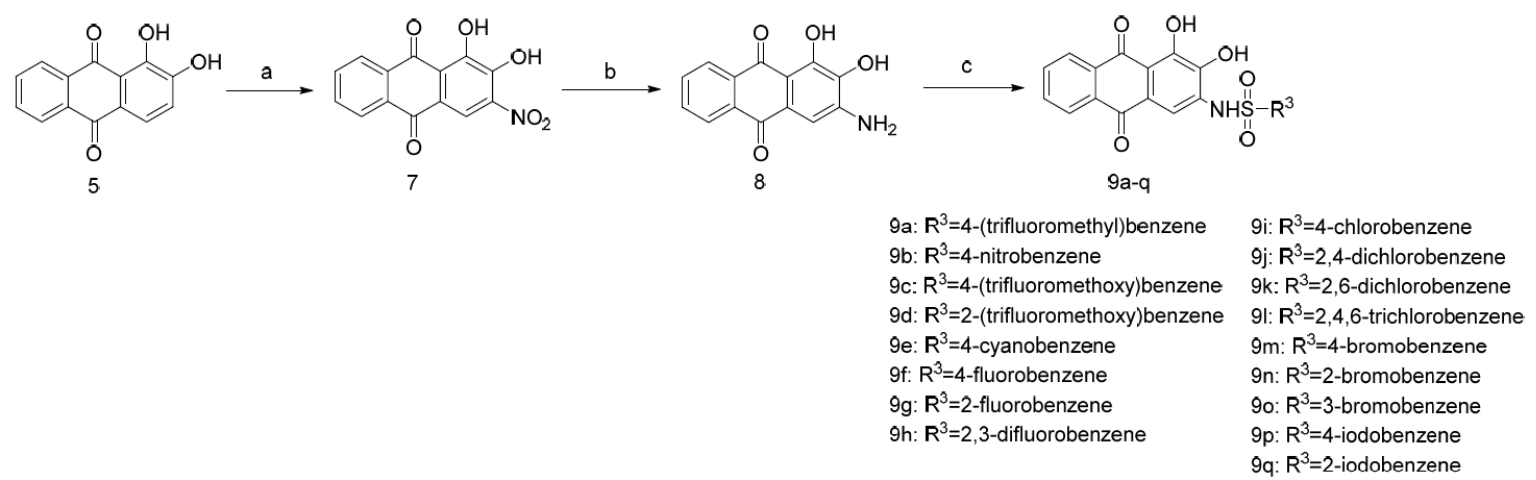

Scheme 2. Synthesis of compounds 9a-q. Reagents and conditions: (a) $\mathrm{HNO}_{3}, \mathrm{CH}_{3} \mathrm{COOH}, 50{ }^{\circ} \mathrm{C}$; (b) $\mathrm{Sn}, \mathrm{SnCl}_{2} \cdot \mathrm{H}_{2} \mathrm{O}, \mathrm{HCl}, \mathrm{EtOH} ;$ (c) $\mathrm{R}^{3} \mathrm{SO}_{2} \mathrm{Cl}, \mathrm{Py}$.

As shown in Scheme 3, the 4-nitro group was reduced to an amino group after the nitrification of compound $\mathbf{6 c}$, followed by sulfonylation of the amino group and hydrolysis of the ester as described above. 

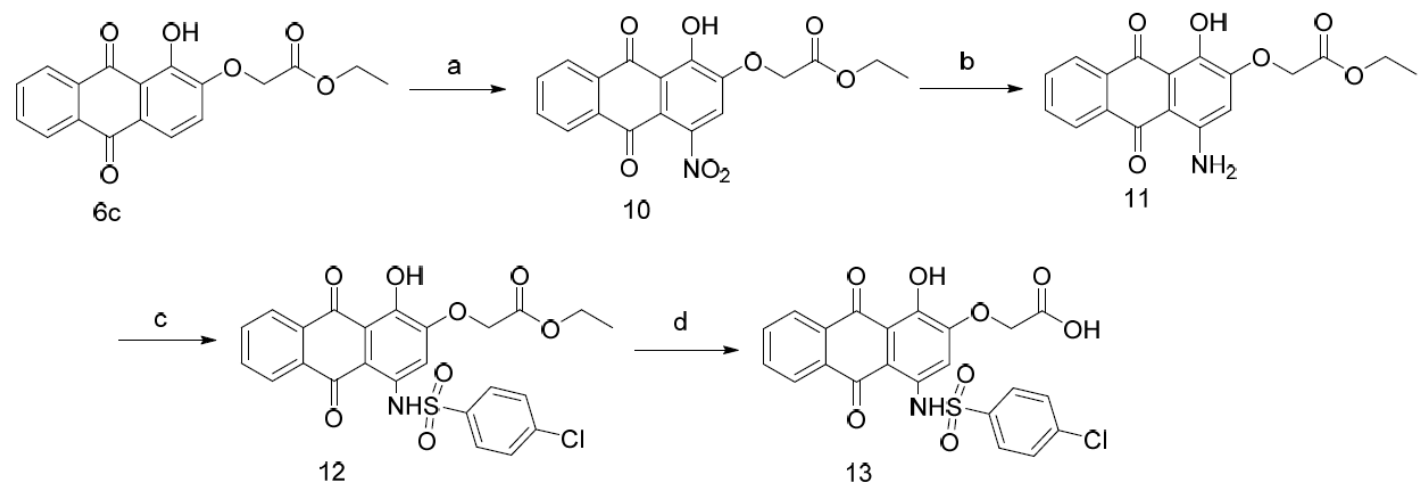

Scheme 3. Synthesis of compounds 12 and 13. Reagents and conditions: (a) $\mathrm{HNO}_{3}, \mathrm{CH}_{3} \mathrm{COOH}, 50{ }^{\circ} \mathrm{C}$; (b) $\mathrm{Sn}, \mathrm{SnCl}_{2} \cdot \mathrm{H}_{2} \mathrm{O}, \mathrm{HCl}, \mathrm{EtOH}$; (c) 4-chlorobenzenesulfonyl chloride, $\mathrm{Py}$; (d) $\mathrm{LiOH}, \mathrm{MeOH}, \mathrm{H}_{2} \mathrm{O}, 30{ }^{\circ} \mathrm{C}$.

Finally, compound $\mathbf{9 i}$ was employed as a starting material, and compounds $\mathbf{1 4 a -} \mathbf{g}$ were obtained by substitution with different reagents and further hydrolysis of the ester or amidation of the carboxyl group (Scheme 4).

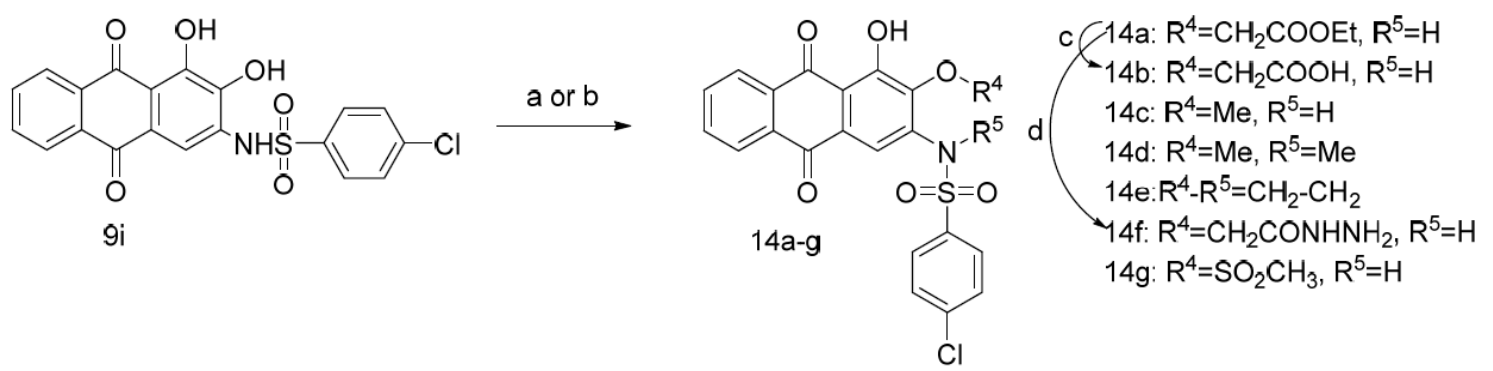

Scheme 4. Synthesis of compounds 14a-g. Reagents and conditions: (a) $\mathrm{K}_{2} \mathrm{CO}_{3}$, DMF, corresponding substituted reagent; (b) methanesulfonyl chloride, DIPEA, Py; (c) $\mathrm{LiOH}, \mathrm{MeOH}, \mathrm{H}_{2} \mathrm{O}, 30{ }^{\circ} \mathrm{C}$; (d) $\mathrm{NH}_{2} \mathrm{NH}_{2} \cdot \mathrm{H}_{2} \mathrm{O}, \mathrm{EtOH}$, reflux.

\subsection{SAR Exploration of the Anthraquinone Derivatives against PGAM1}

Previously we reported several anthraquinone compounds as PGAM1 inhibitors [17]. Due to the limited amount of compounds and the lack of chemical diversity, the SAR of anthraquinone derivatives and PGAM1 remained poorly understood. Here we synthesized four classes of compounds on the base of bioisosterism to study the SAR of anthraquinone derivatives against PGAM1 in detail (Figure 2), using an optimized enzymatic assay in which PGMI-004A served as a control with an $\mathrm{IC}_{50}$ value of $1.2 \pm 0.3 \mu \mathrm{M}$. Firstly, we found that different modifications of the hydroxyl of alizarin (6a-e) showed weak PGAM1 inhibition activity at $10 \mu \mathrm{M}$ (Table 1 ).

In order to explore the SAR of 3-substituents of the anthraquinone scaffold against PGAM1, compounds $\mathbf{9 a}-\mathbf{q}$ were synthesized by reversing the sulfonamide group of PGMI-004A. Compounds 9a-e with different substituents on the phenyl ring displayed comparable inhibition activity towards PGAM1, with $\mathrm{IC}_{50}$ values of $0.5 \mu \mathrm{M}$ or so (Table 2). They were much more potent than the compounds without the sulfonamide group, suggesting the sulfonamide group was essential for maintaining potency. Then, we studied the impact of substituents of different halogens on the phenyl ring (9f-q) on the inhibition activity towards PGAM1 (Table 2). Generally, compounds with $\mathrm{Cl}$, Br and I substituents (9i-q) with $\mathrm{IC}_{50}$ values below $0.5 \mu \mathrm{M}$ performed better than F substituents $(\mathbf{9 f}-\mathbf{h})$. Among them, we discovered one of the most potent PGAM1 inhibitors $9 i$ with an $\mathrm{IC}_{50}$ value of $0.27 \mu \mathrm{M}$ and we solved the co-crystal structure of compound 9i with PGAM1 which would be discussed below. 
Table 1. Inhibition activity of compounds $\mathbf{6 a}-\mathbf{e}$ towards PGAM1.

Compound

\footnotetext{
${ }^{1}$ The data are presented as mean \pm s.d. $(n=2)$.
}

Table 2. Inhibition activity of compounds $\mathbf{9 a}-\mathbf{q}$ towards PGAM1.

Compound


Table 2. Cont.

\begin{tabular}{|c|c|c|}
\hline Compound & $\mathbf{R}^{3}$ & PGAM1 IC $_{50}(\mu \mathrm{M})^{1}$ \\
\hline $9 \mathrm{i}$ & & $0.27 \pm 0.04$ \\
\hline $9 j$ & & $0.28 \pm 0.07$ \\
\hline $9 k$ & & $0.89 \pm 0.33$ \\
\hline 91 & & $0.90 \pm 0.26$ \\
\hline $9 m$ & & $0.26 \pm 0.02$ \\
\hline $9 n$ & & $0.20 \pm 0.04$ \\
\hline 90 & & $0.35 \pm 0.005$ \\
\hline $9 p$ & & $0.47 \pm 0.07$ \\
\hline $9 q$ & & $0.26 \pm 0.03$ \\
\hline
\end{tabular}

${ }^{1}$ The data are presented as mean \pm s.d. $(n=3)$.

We next moved the sulfonamide to the $\mathrm{C} 4$ position of alizarin by substituting the hydroxyl, followed by nitrification, reduction, and sulfonylation. Probably due to the modification of the hydroxyl and replacement of the sulfonamide, compounds 12 and 13 failed to maintain potency, decreasing $10 \%$ and $34 \%$ of PGAM 1 activity at $10 \mu \mathrm{M}$, respectively. Finally, considering the good solubility and potency of inhibitor $9 \mathbf{i}$, we kept the 4-chlorobenzene group and synthesized compounds 14a-g. However, diverse modifications of the hydroxyl caused a loss of inhibition activity toward PGAM1 to different extents (Table 3).

In order to exclude the inhibition of the other three enzymes (enolase-pyruvate kinase-LDH) in the coupled assay, we performed counter screening of selected compounds with $\mathrm{IC}_{50}$ value below $1 \mu \mathrm{M}$ on PGAM1.

As shown in Table 4, the compounds displayed almost no inhibition on the three downstream enzymes at $0.5 \mu \mathrm{M}$ which further confirmed the on-target effect of the compounds. 
Table 3. Inhibition activity of compounds $\mathbf{1 4 a - 1 4 g}$ towards PGAM1.

\begin{tabular}{|c|c|c|c|}
\hline Compound & $R^{5}$ & $R^{6}$ & PGAM1 IC ${ }_{50}(\mu \mathrm{M})^{1}$ \\
\hline $14 a$ & & $\mathrm{H}$ & $>10 \mu \mathrm{M}$ \\
\hline $14 b$ & & $\mathrm{H}$ & $>10 \mu \mathrm{M}$ \\
\hline $14 \mathrm{c}$ & $\mathrm{CH}_{3}$ & $\mathrm{H}$ & $2.92 \pm 0.11$ \\
\hline 14d & $\mathrm{CH}_{3}$ & $\mathrm{CH}_{3}$ & $7.01 \pm 0.92$ \\
\hline $14 \mathrm{e}$ & $-\mathrm{CH}_{2}$ & $-\mathrm{CH}_{2}-$ & $>10 \mu \mathrm{M}$ \\
\hline $14 \mathrm{f}$ & $\mathrm{N} F$ & $\mathrm{H}$ & $2.0 \pm 0.2$ \\
\hline $14 \mathrm{~g}$ & \{ & $\mathrm{H}$ & $>10 \mu \mathrm{M}$ \\
\hline
\end{tabular}

${ }^{1}$ The data are presented as mean \pm s.d. $(n=3)$.

Table 4. The inhibition in the control linked enzyme assay of selected compounds.

\begin{tabular}{ccc}
\hline Compound & PGAM1 IC $_{\mathbf{5 0}}(\boldsymbol{\mu M})$ & $\begin{array}{c}\text { Inhibition in the Control Linked } \\
\text { Enzyme Assay }(\mathbf{0 . 5} \boldsymbol{\mu M})^{\mathbf{1}}\end{array}$ \\
\hline $\mathbf{9 a}$ & $0.54 \pm 0.04$ & $8.0 \pm 5.0 \%$ \\
$\mathbf{9 b}$ & $0.47 \pm 0.08$ & $-2.9 \pm 0.3 \%$ \\
$\mathbf{9 c}$ & $0.90 \pm 0.07$ & $3.7 \pm 1.8 \%$ \\
$\mathbf{9 e}$ & $0.61 \pm 0.12$ & $6.7 \pm 0.2 \%$ \\
$\mathbf{9 f}$ & $0.54 \pm 0.07$ & $5.7 \pm 0.7 \%$ \\
$\mathbf{9 g}$ & $0.79 \pm 0.04$ & $10.2 \pm 3.0 \%$ \\
$\mathbf{9 h}$ & $0.89 \pm 0.13$ & $0.06 \pm 0.4 \%$ \\
$\mathbf{9 i}$ & $0.27 \pm 0.04$ & $-6.4 \pm 5.0 \%$ \\
$\mathbf{9 j}$ & $0.28 \pm 0.07$ & $-2.0 \pm 5.1 \%$ \\
$\mathbf{9 k}$ & $0.89 \pm 0.33$ & $0.5 \pm 3.2 \%$ \\
$\mathbf{9 1}$ & $0.90 \pm 0.26$ & $0.2 \pm 1.7 \%$ \\
$\mathbf{9 m}$ & $0.26 \pm 0.02$ & $-0.1 \pm 2.5 \%$ \\
$\mathbf{9 n}$ & $0.20 \pm 0.04$ & $6.1 \pm 0.8 \%$ \\
$\mathbf{9 0}$ & $0.35 \pm 0.005$ & $7.7 \pm 0.2 \%$ \\
$\mathbf{9 p}$ & $0.47 \pm 0.07$ & $1.9 \pm 0.1 \%$ \\
$\mathbf{9 q}$ & $0.26 \pm 0.03$ & $-2.3 \pm 2.4 \%$ \\
\hline
\end{tabular}

${ }^{1}$ The data are presented as mean \pm s.d. $(n=2)$.

\subsection{Binding Mode of Compound $\mathbf{9 i}$ with PGAM1}

To further understand the molecular mechanism of the anthraquinone derivatives interacting with PGAM1, we determined the X-ray structure of PGAM1 in complex with compound $\mathbf{9} \mathbf{i}$ at resolution of $1.98 \AA$ (Table 5). Compound 9i occupied a novel allosteric site adjacent to substrate binding site with nice electron density (Figure 3A,B). The allosteric pocket was surrounded by the residues of F22, R90, K100, R116 and R191. In detail, the anthraquinone scaffold and sulfonamide of compound 9 i interacted with the main chain carbonyl of K100 through water bridges (Figure 3C). In addition, a hydrophobic interaction was observed between F22 and chlorine-substituted phenyl ring of compound $9 \mathbf{i}$ (Figure 3C). 
Compound $9 \mathbf{i}$ also engaged in a $\pi$-cation interaction with R116 (Figure 3C), which explains why modifications of the hydroxyl group led to decreased potency [39]. To validate the binding mode revealed by the co-crystal structure, we tested the activity of PGAM1 mutants (Supplementary Data, Figure S1) and the inhibition activity of compound $9 \mathbf{i}$ on different mutations of PGAM1. Compound 9i failed to inhibit mutations of PGAM1 (F22A, R116H and R191H) as effectively as the wild type at concentration of $5 \mu \mathrm{M}$ which agreed with the results from crystal structure. Furthermore, a substrate competitive assay demonstrated that compound $9 \mathbf{i}$ held a non-competitive property with substrate 3PG which was also consistent with the binding mode revealed by $\mathrm{X}$-ray structure. The co-crystal structure together with the molecular biological assays illustrated the binding mode of the anthraquinone inhibitor with PGAM1 and provided useful information for further optimization.

Table 5. Data collection and refinement statistics.

\begin{tabular}{|c|c|}
\hline Property & \multirow{2}{*}{ Value } \\
\hline Data Collection & \\
\hline Space group & $\mathrm{P} 2{ }_{1} 2_{1} 2_{1}$ \\
\hline $\begin{array}{c}\text { Cell constants } \\
\text { a, b, c }(\AA)\end{array}$ & $82.54,82.76,104.20$ \\
\hline$\alpha, \beta, \gamma\left({ }^{\circ}\right)$ & $90.00,90.00,90.00$ \\
\hline Resolution * $(\AA)$ & 50-1.98 (2.05-1.98) \\
\hline \% Data completeness & $100.0(100.0)$ \\
\hline No. Observations & 330639 \\
\hline No. Unique Reflections & $50593(4986)$ \\
\hline Redundancy & $6.5(6.6)$ \\
\hline$R_{\text {merge }}$ & $0.06(0.88)$ \\
\hline$<\mathrm{I} / \sigma(\mathrm{I})>$ & 2.05 (at $1.98 \AA$ ) \\
\hline $\mathrm{CC} 1 / 2$ & $0.996(0.728)$ \\
\hline \multicolumn{2}{|l|}{ Refinement } \\
\hline Resolution $(\AA)$ & 38.89-1.98 (2.02-1.98) \\
\hline$\%$ Data completeness & $100.0(100.0)$ \\
\hline No. reflections & $50362(2636)$ \\
\hline $\mathrm{R}_{\text {work }} / \mathrm{R}_{\text {free }}$ & $0.197 / 0.225$ \\
\hline$R_{\text {free }}$ test set & 2569 reflections $(5.08 \%)$ \\
\hline Wilson B-factor $\left(\AA^{2}\right)$ & 39.4 \\
\hline $\mathrm{F}_{\mathrm{O}}, \mathrm{F}_{\mathrm{C}}$ correlation & 0.96 \\
\hline Total number of atoms & 4046 \\
\hline Average $B$, all atoms $\left(\AA^{2}\right)$ & 41.0 \\
\hline Ramachandran favored (\%) & 98.7 \\
\hline Ramachandran allowed (\%) & 1.3 \\
\hline Ramachandran outliers (\%) & 0.0 \\
\hline Rmsd bond lengths $(\AA)$ & 0.007 \\
\hline Rmsd bond angles $\left(^{\circ}\right)$ & 1.044 \\
\hline PDB Accession Code & $6 \mathrm{ISN}$ \\
\hline
\end{tabular}


(a)

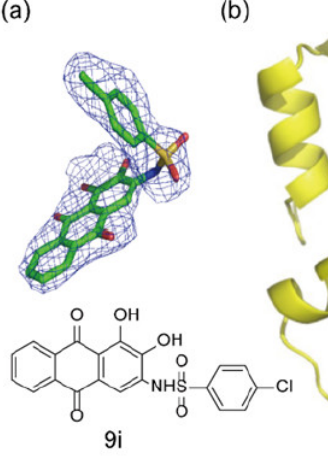

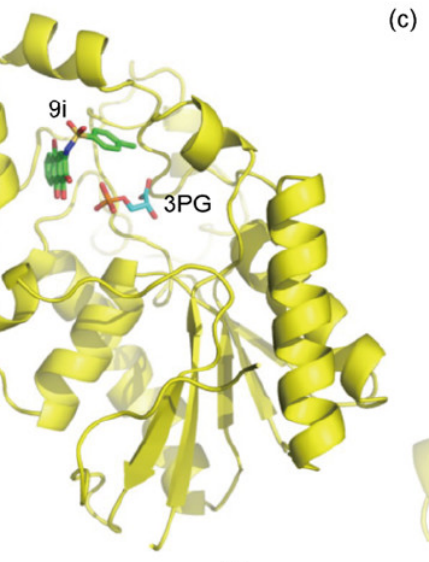

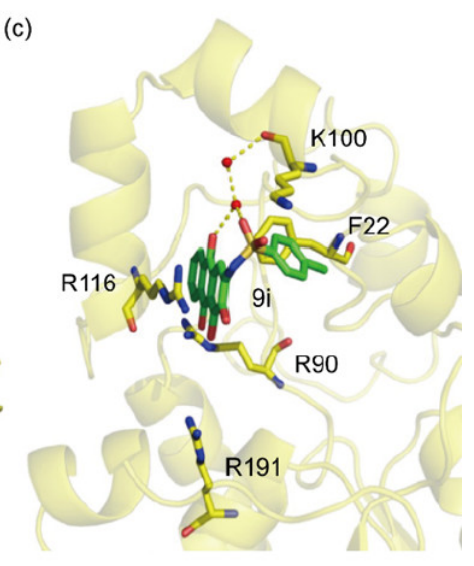

(e)
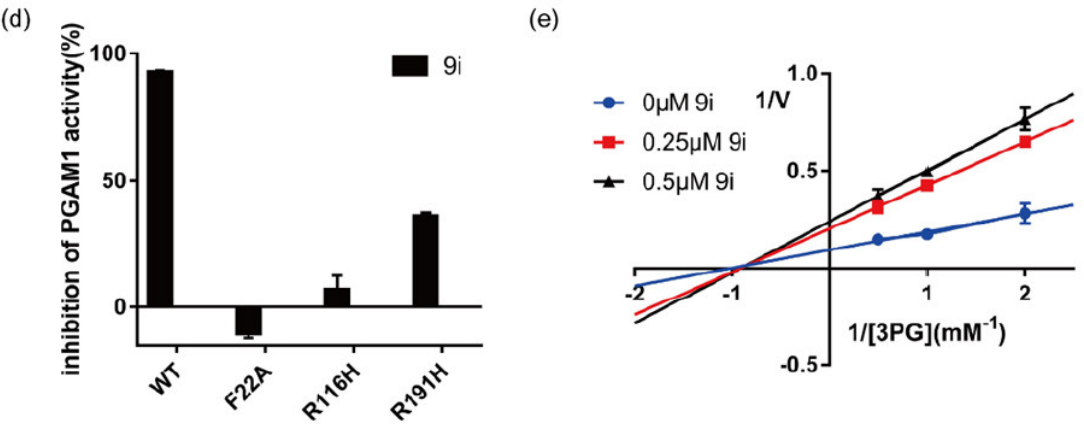

Figure 3. Binding mode of anthraquinone inhibitor 9i with PGAM1. (a) Chemical structure of compound $9 \mathbf{i}$ and Fo-Fc electron density of compound $9 \mathbf{i}$ contoured at 2.0 $\sigma$; (b) Overlay of compound 9i (PBD: 6ISN) and 3PG (PBD:2F90) in PGAM1; (c) Interactions of compound 9i and the critical residues of PGAM1 in the co-crystal structure; (d) Inhibition of compounds $9 \mathbf{i}$ on wild-type and mutations of PGAM1 at concentration of $5 \mu \mathrm{M}$; (e) Noncompetitive property of compound $9 \mathbf{i}$ with substrate 3PG. The data are presented as mean \pm s.d.

\subsection{Inhibition Activity of Selected Compounds on Cancer Cell Proliferation}

Given PGAM1 plays a crucial part in cancer metabolism, inhibition of PGAM1 by small molecules is supposed to suppress cancer cell proliferation. We selected the compounds with $\mathrm{IC}_{50}$ values below $10 \mu \mathrm{M}$ toward PGAM1 to inhibit H1299 cells proliferation. Then the potent inhibitors with $\mathrm{IC}_{50}$ values below $20 \mu \mathrm{M}$ on H1299 cells were further tested on A549 and PC9 cells. In general, proliferation inhibition of the compounds was correlated with PGAM1 inhibition and the inhibitors performed similarly among different cancer cells, with $\mathrm{IC}_{50}$ values ranging from approximately 6-50 $\mu \mathrm{M}$ (Tables 6 and 7). Notably, compound $9 \mathrm{i}$ effectively suppressed the three different cancer cells proliferation which was also potent towards PGAM1. 
Table 6. Inhibition activity of compounds $\mathbf{9 a}-\mathbf{q}$ on cancer cell proliferation.

\begin{tabular}{|c|c|c|c|c|}
\hline Compound & $\mathbf{R}^{3}$ & H1299 IC $50(\mu \mathrm{M})$ & A549 $\mathrm{IC}_{50}(\mu \mathrm{M})$ & PC9 IC $_{50}(\mu \mathrm{M})^{1}$ \\
\hline $9 a$ & & $20.7 \pm 0.6$ & n.d. & n.d. \\
\hline $9 b$ & & $25.8 \pm 2.2$ & n.d. & n.d. \\
\hline $9 c$ & & $16.6 \pm 1.8$ & $8.0 \pm 0.8$ & $10.2 \pm 0.7$ \\
\hline $9 d$ & & $13.9 \pm 3.6$ & $17.0 \pm 4.9$ & $44.2 \pm 1.5$ \\
\hline $9 e$ & & $26.3 \pm 4.5$ & n.d. & n.d. \\
\hline $9 f$ & & $24.2 \pm 2.6$ & n.d. & n.d. \\
\hline $9 g$ & & $31.3 \pm 1.9$ & n.d. & n.d. \\
\hline $9 h$ & & $45.7 \pm 2.7$ & n.d. & n.d. \\
\hline $9 \mathrm{i}$ & & $6.9 \pm 1.2$ & $12.7 \pm 2.7$ & $13.8 \pm 1.0$ \\
\hline $9 j$ & & $23.7 \pm 1.1$ & n.d. & n.d. \\
\hline $9 k$ & & $34.6 \pm 2.4$ & n.d. & n.d. \\
\hline 91 & & $6.2 \pm 1.2$ & $>50$ & $>50$ \\
\hline $9 m$ & & $28.1 \pm 1.9$ & n.d. & n.d. \\
\hline $9 n$ & & $50 \pm 0.6$ & n.d. & n.d. \\
\hline 90 & & $24.5 \pm 0.2$ & n.d. & n.d. \\
\hline $9 p$ & & $17.3 \pm 3.9$ & $10.3 \pm 1.4$ & $13.4 \pm 1.8$ \\
\hline $9 q$ & & $26.4 \pm 3.8$ & n.d. & n.d. \\
\hline
\end{tabular}

\footnotetext{
${ }^{1}$ The data are presented as mean \pm s.d. $(\mathrm{n}=3)$ n.d. $=$ not determined.
} 
Table 7. Inhibition activity of compounds $\mathbf{1 4 a}-\mathbf{g}$ on cancer cell proliferation.

\begin{tabular}{|c|c|c|c|c|c|}
\hline Compound & $\mathbf{R}^{5}$ & $\mathbf{R}^{6}$ & $\mathrm{H} 1299 \mathrm{IC}_{50}(\mu \mathrm{M})$ & A549 $\mathrm{IC}_{50}(\mu \mathrm{M})$ & PC9 $\mathrm{IC}_{50}(\mu \mathrm{M})^{1}$ \\
\hline $14 \mathrm{c}$ & $\mathrm{CH}_{3}$ & $\mathrm{H}$ & $26.9 \pm 2.1$ & n.d. & n.d. \\
\hline $14 d$ & $\begin{array}{l}\mathrm{CH}_{3} \\
\mathrm{O}\end{array}$ & $\mathrm{CH}_{3}$ & $10.3 \pm 0.8$ & $9.9 \pm 0.2$ & $49.1 \pm 1.9$ \\
\hline $14 f$ & $\mathrm{NF}$ & $\mathrm{H}$ & $>100$ & n.d. & n.d. \\
\hline $14 \mathrm{~g}$ & s & $\mathrm{H}$ & $28.5 \pm 0.6$ & n.d. & n.d. \\
\hline
\end{tabular}

\footnotetext{
${ }^{1}$ The data are presented as mean \pm s.d. $(\mathrm{n}=3)$ n.d. $=$ not determined.
}

\section{Materials and Methods}

\subsection{General Procedures}

All reagents were purchased commercially. ${ }^{1} \mathrm{H}-\mathrm{NMR}$ and ${ }^{13} \mathrm{C}-\mathrm{NMR}$ spectra were recorded on Bruker AC400 and Bruker AC600 NMR spectrometers, respectively (Billerica, MA, USA). Low-resolution mass spectra were recorded on a 6120 Quadrupole mass spectrometer (Agilent, Santa Clara, CA, USA) equipped with electrospray ionization (ESI). High-resolution mass spectra were determined on triple TOF $5600^{+}$MS /MS system (AB Sciex, Concord, ON, Canada) in negative ESI mode. The purity of target compounds was determined by high-performance liquid chromatography (Agilent, Santa Clara, CA, USA, DIKMA Diamonsil Plus C18, $250 \times 4.6 \mathrm{~mm}, 5 \mu \mathrm{m}, 25^{\circ} \mathrm{C}$, UV $290 \mathrm{nM}$ ). All the biologically tested compounds achieved $\geq 95 \%$ purity.

\subsection{Synthesis of diethyl 2,2'-((9,10-dioxo-9,10-dihydroanthracene-1,2-diyl) bis(oxy)) diacetate (6a)}

1,2-Dihydroxyanthracene-9,10-dione (1.2 g, $5 \mathrm{mmol})$, ethyl 2-bromoacetate $(2 \mathrm{~g}, 12 \mathrm{mmol})$ and $\mathrm{K}_{2} \mathrm{CO}_{3}(1.66 \mathrm{~g}, 12 \mathrm{mmol}$ ) were added to $\mathrm{N}, \mathrm{N}$-dimethylformamide (DMF, $50 \mathrm{~mL}$ ) and the mixture was stirred at $80{ }^{\circ} \mathrm{C}$. The mixture was cooled to room temperature and poured into $10 \%$ aqueous $\mathrm{HCl}$ $(350 \mathrm{~mL})$. Then the suspension was extracted with ethyl acetate and the organic phase was washed with brine, dried over anhydrous $\mathrm{Na}_{2} \mathrm{SO}_{4}$ and filtered. The residue was purified by silica chromatography after removal of ethyl acetate to afford $6 \mathrm{a}$ as a yellow solid $(0.89 \mathrm{~g}, 43 \%) .{ }^{1} \mathrm{H}-\mathrm{NMR}\left(400 \mathrm{MHz}, \mathrm{DMSO}-d_{6}\right)$ $\delta 8.16-8.10(\mathrm{~m}, 2 \mathrm{H}), 8.01(\mathrm{~d}, J=8.8 \mathrm{~Hz}, 1 \mathrm{H}), 7.93-7.84(\mathrm{~m}, 2 \mathrm{H}), 7.53(\mathrm{~d}, J=8.8 \mathrm{~Hz}, 1 \mathrm{H}), 5.07(\mathrm{~s}, 2 \mathrm{H}), 4.74$ $(\mathrm{s}, 2 \mathrm{H}), 4.20(\mathrm{qd}, J=4.0,7.2 \mathrm{~Hz}, 4 \mathrm{H}), 1.23(\mathrm{td}, J=2.4,7.2 \mathrm{~Hz}, 6 \mathrm{H}) .{ }^{13} \mathrm{C}-\mathrm{NMR}(151 \mathrm{MHz}, \mathrm{DMSO}) \delta 181.69$, 181.42, 168.27, 167.97, 156.49, 146.23, 134.58, 134.32, 133.94, 132.26, 127.15, 126.94, 126.67, 126.18, 124.64, 118.20, 68.72, 65.22, 60.99, 60.44, 14.07, 13.98. MS (ESI) $(m / z): 413.1(\mathrm{M}-\mathrm{H})^{-}$. HRMS (ESI) calcd. for $\mathrm{C}_{22} \mathrm{H}_{20} \mathrm{O}_{8}[\mathrm{M}-\mathrm{H}]^{-}:$413.1231; found: 413.1239 . Purity $95.1 \%$ by HPLC.

\subsection{Synthesis of 2,2'-((9,10-dioxo-9,10-dihydroanthracene-1,2-diyl) bis(oxy)) diacetic acid (6b)}

Compound $6 \mathrm{a}(206 \mathrm{mg}, 0.5 \mathrm{mmol})$ and $0.5 \mathrm{M}$ aqueous $\mathrm{LiOH}(8 \mathrm{~mL})$ were added into methanol $(8 \mathrm{~mL})$ and the mixture was stirred at $30^{\circ} \mathrm{C}$. The mixture was added dropwise to $10 \%$ aqueous $\mathrm{HCl}$ and acidified to $\mathrm{pH} 1-2$. A yellow solid $(169 \mathrm{mg}, 95 \%)$ of $6 \mathrm{~b}$ was obtained by filtration. ${ }^{1} \mathrm{H}-\mathrm{NMR}$ $\left(400 \mathrm{MHz}, \mathrm{DMSO}-d_{6}\right) \delta 12.76(\mathrm{brs}, 2 \mathrm{H}), 8.14(\mathrm{~d}, J=7.6 \mathrm{~Hz}, 2 \mathrm{H}), 8.01(\mathrm{~d}, J=8.4 \mathrm{~Hz}, 1 \mathrm{H}), 7.93-7.85(\mathrm{~m}$, 2H), $7.51(\mathrm{~d}, J=8.8 \mathrm{~Hz}, 1 \mathrm{H}), 4.98(\mathrm{~s}, 2 \mathrm{H}), 4.67(\mathrm{~s}, 2 \mathrm{H}) .{ }^{13} \mathrm{C}-\mathrm{NMR}(151 \mathrm{MHz}, \mathrm{DMSO}) \delta 181.95,181.45$, $169.75,169.45,156.76,146.32,134.62,134.31$, 133.98, 132.34, 126.97, 126.82, 126.71, 126.20, 124.61, 118.12, 
68.62, 65.05. MS (ESI) $(m / z): 355.0(\mathrm{M}-\mathrm{H})^{-}$. HRMS (ESI) calcd. for $\mathrm{C}_{18} \mathrm{H}_{12} \mathrm{O}_{8}[\mathrm{M}-\mathrm{H}]^{-}$: 355.0459 ; found: 355.0462 . Purity $95.1 \%$ by HPLC.

\subsection{Synthesis of 2-((1-hydroxy-9,10-dioxo-9,10-dihydroanthracen-2-yl) oxy)-N,N-dimethylacetamide (6e)}

Compound 6d (149 mg, $0.5 \mathrm{mmol})$, dimethylamine hydrochloride (31 mg, $0.68 \mathrm{mmol})$, $N$-(3-dimethylaminopropyl)- $N^{\prime}$-ethylcarbodiimide hydrochloride (EDCI, $120 \mathrm{mg}, 0.6 \mathrm{mmol}$ ), 1-hydroxybenzotriazole (HOBt, $80 \mathrm{mg}, 0.6 \mathrm{mmol}$ ) and diisopropylethylamine (DIPEA, $175 \mu \mathrm{L}, 1 \mathrm{mmol}$ ) were added to dry DMF $(10 \mathrm{~mL})$ and the mixture was stirred at room temperature. The mixture was added dropwise to $\mathrm{H}_{2} \mathrm{O}(140 \mathrm{~mL})$. An orange solid $(114 \mathrm{mg}, 70 \%)$ of $6 \mathrm{e}$ was obtained by filtration. ${ }^{1} \mathrm{H}-\mathrm{NMR}\left(400 \mathrm{MHz}\right.$, DMSO- $\left.d_{6}\right) \delta 12.74(\mathrm{~s}, 1 \mathrm{H}), 8.28-8.11(\mathrm{~m}, 2 \mathrm{H}), 7.99-7.87(\mathrm{~m}, 2 \mathrm{H}), 7.68(\mathrm{~d}, J=8.4 \mathrm{~Hz}$, $1 \mathrm{H}), 7.31(\mathrm{~d}, J=8.8 \mathrm{~Hz}, 1 \mathrm{H}), 5.09(\mathrm{~s}, 2 \mathrm{H}), 3.02(\mathrm{~s}, 3 \mathrm{H}), 2.87(\mathrm{~s}, 3 \mathrm{H}) .{ }^{13} \mathrm{C}-\mathrm{NMR}(151 \mathrm{MHz}, \mathrm{DMSO}) \delta 188.67$, 180.76, 166.18, 152.60, 151.79, 135.20, 134.26, 133.48, 132.95, 126.81, 126.60, 124.89, 120.20, 118.29, 115.94, 66.12, 35.46, 35.01. MS (ESI) $(m / z): 324.0(\mathrm{M}-\mathrm{H})^{-}$. HRMS (ESI) calcd. for $\mathrm{C}_{18} \mathrm{H}_{15} \mathrm{NO}_{5}[\mathrm{M}-\mathrm{H}]^{-}$: 324.0877; found: 324.0876 . Purity $96.4 \%$ by HPLC.

\subsection{General Procedure for the Synthesis of Compounds $\mathbf{9 a}-\mathbf{q}$}

Step 1. Nitric acid (1.5 mL, 33.33 mmol, Sinopharm Chemical Reagent Co., Ltd, Shanghai, China) was added to the suspension of alizarin $(5 \mathrm{~g}, 20.8 \mathrm{mmol})$ in acetic acid $(350 \mathrm{~mL})$ at $50{ }^{\circ} \mathrm{C}$. A yellow solid (4.15 g, 70\%) of crude product 6 was obtained by filtration [40].

Step 2. Sn (10.5 g, $341 \mathrm{mmol}$, Sinopharm Chemical Reagent Co., Ltd, Shanghai, China), $\mathrm{SnCl}_{2} \cdot 2 \mathrm{H}_{2} \mathrm{O}(12.5 \mathrm{~g}, 55.4 \mathrm{mmol})$ and concentrated $\mathrm{HCl}(50.4 \mathrm{~mL}, 604.8 \mathrm{mmol})$ were added to the suspension of crude product $6(1.75 \mathrm{~g}, 6.14 \mathrm{mmol})$ in ethanol $(350 \mathrm{~mL}$, Sinopharm) and the mixture was stirred at room temperature overnight. Then the mixture was poured into water $(1 \mathrm{~L})$ and a red solid precipitated. A black solid of crude product $7(1.41 \mathrm{~g}, 90 \%)$ was obtained after filtration and being dried under vacuum [40].

Step 3. To the solution of crude product 7 ( $255 \mathrm{mg}, 1 \mathrm{mmol})$ in dry pyridine ( $5 \mathrm{~mL}$, Sinopharm), corresponding sulfonyl chloride ( $1.5 \mathrm{mmol}$, Energy Chemical, Shanghai, China) was added and the mixture was stirred at room temperature. Then it was added to $10 \%$ aqueous $\mathrm{HCl}$ ( $50 \mathrm{~mL}$, Sinopharm) and the suspension was extracted with ethyl acetate (Sinopharm). The organic phase was washed with brine, dried over anhydrous $\mathrm{Na}_{2} \mathrm{SO}_{4}$ and filtered. The residue was purified by silica chromatography after removal of ethyl acetate to give compounds $\mathbf{9 a}-\mathbf{q}$.

N-(3,4-Dihydroxy-9,10-dioxo-9,10-dihydroanthracen-2-yl)-4-(trifluoromethyl) benzenesulfonamide (9a). Yellow solid, 25\% yield. ${ }^{1} \mathrm{H}-\mathrm{NMR}\left(400 \mathrm{MHz}\right.$, DMSO- $\left.d_{6}\right) \delta 12.60$ (brs, $\left.1 \mathrm{H}\right), 10.90$ (brs, $1 \mathrm{H}$ ), 10.67 (brs, $1 \mathrm{H}), 8.22-8.12(\mathrm{~m}, 2 \mathrm{H}), 8.09(\mathrm{~d}, J=8.4 \mathrm{~Hz}, 2 \mathrm{H}), 8.01(\mathrm{~d}, J=8.4 \mathrm{~Hz}, 2 \mathrm{H}), 7.95-7.86(\mathrm{~m}, 2 \mathrm{H}), 7.73(\mathrm{~s}, 1 \mathrm{H})$. ${ }^{13} \mathrm{C}-\mathrm{NMR}(151 \mathrm{MHz}, \mathrm{DMSO}) \delta 187.78,180.56,150.37,144.20,143.23,135.02,134.22,133.28,132.79$, $132.68(\mathrm{q}, J=31.7 \mathrm{~Hz}), 130.34,127.60(2 \mathrm{C}), 126.77,126.61,126.59,126.39,123.71,123.38(\mathrm{q}, J=273.3 \mathrm{~Hz})$, 113.49, 113.35. MS (ESI) $(\mathrm{m} / z): 462.0(\mathrm{M}-\mathrm{H})^{-}$. HRMS (ESI) calcd. for $\mathrm{C}_{21} \mathrm{H}_{12} \mathrm{~F}_{3} \mathrm{NO}_{6} \mathrm{~S}[\mathrm{M}-\mathrm{H}]^{-}$: 462.0265; found: 462.0284 . Purity $99.8 \%$ by HPLC.

$\mathrm{N}$-(3,4-Dihydroxy-9,10-dioxo-9,10-dihydroanthracen-2-yl)-4-nitrobenzenesulfonamide (9b). Orange solid, 50\% yield. ${ }^{1} \mathrm{H}-\mathrm{NMR}\left(400 \mathrm{MHz}\right.$, DMSO- $\left.d_{6}\right) \delta 12.61$ (brs, $\left.1 \mathrm{H}\right), 10.98$ (brs, $\left.1 \mathrm{H}\right), 10.79$ (brs, $\left.1 \mathrm{H}\right), 8.41$ $(\mathrm{d}, J=8.0 \mathrm{~Hz}, 2 \mathrm{H}), 8.25-8.06(\mathrm{~m}, 4 \mathrm{H}), 7.97-7.86(\mathrm{~m}, 2 \mathrm{H}), 7.73(\mathrm{~s}, 1 \mathrm{H}) .{ }^{13} \mathrm{C}-\mathrm{NMR}(151 \mathrm{MHz}, \mathrm{DMSO}) \delta$ $187.79,180.53,150.41,149.90,145.71,143.61,135.05,134.24,133.27,132.79,130.11,128.22(2 \mathrm{C}), 126.77$, 126.41, 124.65(2C), 123.70, 114.04, 113.50. MS (ESI) $(m / z): 439.0(\mathrm{M}-\mathrm{H})^{-}$. HRMS (ESI) calcd. for $\mathrm{C}_{20} \mathrm{H}_{12} \mathrm{~N}_{2} \mathrm{O}_{8} \mathrm{~S}[\mathrm{M}-\mathrm{H}]^{-}:$439.0242; found: 439.0228 . Purity $98.4 \%$ by HPLC.

$\mathrm{N}$-(3,4-Dihydroxy-9,10-dioxo-9,10-dihydroanthracen-2-yl)-4-(trifluoromethoxy) benzenesulfonamide (9c). Yellow solid, $41 \%$ yield. ${ }^{1} \mathrm{H}-\mathrm{NMR}\left(400 \mathrm{MHz}\right.$, DMSO- $\left.d_{6}\right) \delta 12.61$ (brs, $\left.1 \mathrm{H}\right), 10.94$ (brs, $\left.1 \mathrm{H}\right), 10.51$ (brs, $1 \mathrm{H}), 8.22-8.12(\mathrm{~m}, 2 \mathrm{H}), 8.05-7.99(\mathrm{~m}, 2 \mathrm{H}), 7.95-7.85(\mathrm{~m}, 2 \mathrm{H}), 7.74(\mathrm{~s}, 1 \mathrm{H}), 7.61(\mathrm{dd}, J=1.2,9.2 \mathrm{~Hz}, 2 \mathrm{H})$. ${ }^{13} \mathrm{C}-\mathrm{NMR}(151 \mathrm{MHz}$, DMSO) $\delta$ 187.78, 180.60, 151.20, 150.35, 142.96, 139.19, 135.02, 134.23, 133.30, $132.82,130.63,129.29,126.78,126.40,123.74,121.47,119.80$ (q, $J=259.7 \mathrm{~Hz}), 113.22,113.12$. MS (ESI) 
$(m / z): 478.0(\mathrm{M}-\mathrm{H})^{-}$. HRMS (ESI) calcd. for $\mathrm{C}_{21} \mathrm{H}_{12} \mathrm{~F}_{3} \mathrm{NO}_{7} \mathrm{~S}[\mathrm{M}-\mathrm{H}]^{-}$: 478.0214; found: 478.0207. Purity $96.8 \%$ by HPLC.

N-(3,4-Dihydroxy-9,10-dioxo-9,10-dihydroanthracen-2-yl)-2-(trifluoromethoxy) benzenesulfonamide (9d). Yellow solid, $40 \%$ yield. ${ }^{1} \mathrm{H}-\mathrm{NMR}\left(400 \mathrm{MHz}\right.$, DMSO- $\left.d_{6}\right) \delta 12.62$ (brs, $\left.1 \mathrm{H}\right), 11.00$ (brs, $\left.1 \mathrm{H}\right), 10.37$ (brs, $1 \mathrm{H}), 8.24-8.09(\mathrm{~m}, 2 \mathrm{H}), 8.00(\mathrm{dd}, J=2.0,8.0 \mathrm{~Hz}, 1 \mathrm{H}), 7.96-7.86(\mathrm{~m}, 2 \mathrm{H}), 7.83-7.75(\mathrm{~m}, 1 \mathrm{H}), 7.71(\mathrm{~s}$, 1H), 7.63-7.51 (m, 2H). ${ }^{13} \mathrm{C}-\mathrm{NMR}(151 \mathrm{MHz}, \mathrm{DMSO}) \delta 187.81,180.54,150.37,145.18,143.20,135.55$, 135.05, 134.25, 133.28, 132.82, 132.34, 130.65, 130.41, 127.45, 126.80, 126.43, 123.61, 120.97, 119.81 (q, $J=259.7 \mathrm{~Hz}), 113.72,113.26$. MS (ESI) $(\mathrm{m} / z): 477.8(\mathrm{M}-\mathrm{H})^{-}$. HRMS (ESI) calcd. for $\mathrm{C}_{21} \mathrm{H}_{12} \mathrm{~F}_{3} \mathrm{NO}_{7} \mathrm{~S}$ $[\mathrm{M}-\mathrm{H}]^{-}:$478.0214; found: 478.0225 . Purity $95.2 \%$ by HPLC.

4-Cyano-N-(3,4-dihydroxy-9,10-dioxo-9,10-dihydroanthracen-2-yl) benzenesulfonamide (9e). Red solid, $60 \%$ yield. ${ }^{1} \mathrm{H}-\mathrm{NMR}\left(400 \mathrm{MHz}\right.$, DMSO- $\left.d_{6}\right) \delta 12.60$ (brs, $\left.1 \mathrm{H}\right), 10.77$ (brs, $\left.2 \mathrm{H}\right), 8.22-8.12(\mathrm{~m}, 2 \mathrm{H}), 8.09(\mathrm{~d}$, $J=8.0 \mathrm{~Hz}, 2 \mathrm{H}), 8.02(\mathrm{~d}, J=8.4 \mathrm{~Hz}, 2 \mathrm{H}), 7.96-7.87(\mathrm{~m}, 2 \mathrm{H}), 7.70(\mathrm{~s}, 1 \mathrm{H}) .{ }^{13} \mathrm{C}-\mathrm{NMR}(151 \mathrm{MHz}, \mathrm{DMSO}) \delta$ $187.81,180.57,150.39,144.34,143.46,135.05,134.25,133.49,133.29,132.81,130.22,127.34,126.81,126.75$, 126.44, 123.71, 117.60, 115.48, 113.78(2C), 113.44. MS (ESI) $(m / z): 419.0(\mathrm{M}-\mathrm{H})^{-}$. HRMS (ESI) calcd. for $\mathrm{C}_{21} \mathrm{H}_{12} \mathrm{~N}_{2} \mathrm{O}_{6} \mathrm{~S}[\mathrm{M}-\mathrm{H}]^{-}$: 419.0343; found: 419.0348 . Purity $95.1 \%$ by HPLC.

$\mathrm{N}$-(3,4-Dihydroxy-9,10-dioxo-9,10-dihydroanthracen-2-yl)-4-fluorobenzenesulfonamide (9f). Yellow solid, $62 \%$ yield. ${ }^{1} \mathrm{H}-\mathrm{NMR}\left(400 \mathrm{MHz}\right.$, DMSO- $\left.d_{6}\right) \delta 12.58$ (brs, $\left.1 \mathrm{H}\right), 10.84$ (brs, $\left.1 \mathrm{H}\right), 10.43$ (brs, $1 \mathrm{H}$ ), 8.19-8.11 (m, 2H), 8.01-7.84 (m, 4H), $7.73(\mathrm{~s}, 1 \mathrm{H}), 7.46(\mathrm{t}, J=8.8 \mathrm{~Hz}, 2 \mathrm{H}) .{ }^{13} \mathrm{C}-\mathrm{NMR}(151 \mathrm{MHz}, \mathrm{DMSO})$ $\delta 187.75,180.59,164.47(\mathrm{~d}, J=250.7 \mathrm{~Hz}), 150.32,142.76,136.58,134.99,134.21,133.28,132.81,130.82$, $129.75(\mathrm{~d}, J=9.1 \mathrm{~Hz}, 2 \mathrm{C}), 126.76,126.38,123.72,116.57(\mathrm{~d}, J=22.7 \mathrm{~Hz}, 2 \mathrm{C}), 113.10,112.89$. MS (ESI) $(m / z): 412.0(\mathrm{M}-\mathrm{H})^{-}$. HRMS (ESI) calcd. for $\mathrm{C}_{20} \mathrm{H}_{12} \mathrm{FNO}_{6} \mathrm{~S}[\mathrm{M}-\mathrm{H}]^{-}:$412.0297; found: 412.0305 . Purity $98.7 \%$ by HPLC.

N-(3,4-Dihydroxy-9,10-dioxo-9,10-dihydroanthracen-2-yl)-2-fluorobenzenesulfonamide (9g). Yellow solid, 42\% yield. ${ }^{1} \mathrm{H}-\mathrm{NMR}\left(400 \mathrm{MHz}, \mathrm{DMSO}-d_{6}\right) \delta 12.61$ (brs, $\left.1 \mathrm{H}\right), 10.95$ (brs, $\left.1 \mathrm{H}\right), 10.49$ (brs, $1 \mathrm{H}$ ), 8.21-8.09 (m, 2H), 7.95-7.80 (m, 3H), 7.77-7.67 (m, 2H), 7.46 (t, $J=9.4 \mathrm{~Hz}, 1 \mathrm{H}), 7.38(\mathrm{t}, J=7.6 \mathrm{~Hz}, 1 \mathrm{H})$. ${ }^{13} \mathrm{C}-\mathrm{NMR}(151 \mathrm{MHz}, \mathrm{DMSO}) \delta 187.80,180.56,158.34(\mathrm{~d}, J=256.7 \mathrm{~Hz}), 150.40,143.42,136.02,135.01$, 134.21, 133.28, 132.81, 130.39, 129.87, 127.94 (d, J = 12.1 Hz), 126.77, 126.41, 124.90, 123.57, 117.44 (d, $J=21.1 \mathrm{~Hz}), 113.88,113.32$. MS (ESI) $(\mathrm{m} / \mathrm{z}): 412.0(\mathrm{M}-\mathrm{H})^{-}$. HRMS (ESI) calcd. for $\mathrm{C}_{20} \mathrm{H}_{12} \mathrm{FNO}_{6} \mathrm{~S}$ $[\mathrm{M}-\mathrm{H}]^{-}$: 412.0297; found: 412.0300 . Purity $96.1 \%$ by HPLC.

$\mathrm{N}$-(3,4-Dihydroxy-9,10-dioxo-9,10-dihydroanthracen-2-yl)-2,3-difluorobenzenesulfonamide (9h). Red solid, $52 \%$ yield. ${ }^{1} \mathrm{H}-\mathrm{NMR}\left(400 \mathrm{MHz}\right.$, DMSO- $\left.d_{6}\right) \delta 12.60$ (brs, $\left.1 \mathrm{H}\right), 10.83$ (brs, $\left.2 \mathrm{H}\right), 8.23-8.11(\mathrm{~m}, 2 \mathrm{H})$, 7.96-7.86 (m, 2H), $7.79(\mathrm{q}, J=8.6 \mathrm{~Hz}, 1 \mathrm{H}), 7.69(\mathrm{~s}, 1 \mathrm{H}), 7.62(\mathrm{t}, J=7.0 \mathrm{~Hz}, 1 \mathrm{H}), 7.44-7.34(\mathrm{~m}, 1 \mathrm{H})$. ${ }^{13} \mathrm{C}-\mathrm{NMR}(151 \mathrm{MHz}, \mathrm{DMSO}) \delta$ 187.86, 180.52, 150.46, 149.96 (dd, J = 249.9 Hz, J=12.1 Hz), 146.64 (dd, $J=256.7 \mathrm{~Hz}, J=13.6 \mathrm{~Hz}) 144.45,135.05,134.22,133.29,132.81,130.25(\mathrm{~d}, J=10.6 \mathrm{~Hz}), 129.75,126.78(\mathrm{~d}$, $J=10.6 \mathrm{~Hz}), 126.43 \mathrm{~d}, \mathrm{~J}=7.6 \mathrm{~Hz}), 125.26,124.95,123.51,122.86(\mathrm{~d}, J=16.6 \mathrm{~Hz}), 115.28(\mathrm{~d}, J=6.0 \mathrm{~Hz})$, 113.72. MS (ESI) $(m / z): 430.0(\mathrm{M}-\mathrm{H})^{-}$. HRMS (ESI) calcd. for $\mathrm{C}_{20} \mathrm{H}_{11} \mathrm{FNO}_{6} \mathrm{~S}[\mathrm{M}-\mathrm{H}]^{-}:$430.0202; found: 430.0206 . Purity $95.4 \%$ by HPLC.

4-Chloro-N-(3,4-dihydroxy-9,10-dioxo-9,10-dihydroanthracen-2-yl) benzenesulfonamide (9i). Yellow solid, 56\% yield. ${ }^{1} \mathrm{H}-\mathrm{NMR}\left(400 \mathrm{MHz}\right.$, DMSO- $\left.d_{6}\right) \delta 12.61$ (brs, $\left.1 \mathrm{H}\right), 10.94$ (brs, $\left.1 \mathrm{H}\right), 10.47$ (brs, $1 \mathrm{H}$ ), 8.22-8.11 (m, 2H), 7.96-7.83 (m, 4H), 7.78-7.63 (m, 3H). ${ }^{13} \mathrm{C}-\mathrm{NMR}$ (151 MHz, DMSO) $\delta 187.76,180.58$, 150.34, 142.90, 139.07, 138.04, 135.00, 134.22, 133.28, 132.80, 130.63, 129.50(2C), 128.57(2C), 126.77, 126.39, 123.72, 113.18, 113.08. MS (ESI) $(m / z): 427.8(\mathrm{M}-\mathrm{H})^{-}$. HRMS (ESI) calcd. for $\mathrm{C}_{20} \mathrm{H}_{12} \mathrm{ClNO}_{6} \mathrm{~S}$ $[\mathrm{M}-\mathrm{H}]^{-}:$: 428.0001; found: 428.0005 . Purity $99.4 \%$ by HPLC.

2,4-Dichloro-N-(3,4-dihydroxy-9,10-dioxo-9,10-dihydroanthracen-2-yl) benzenesulfonamide (9j). Orange solid, $60 \%$ yield. ${ }^{1} \mathrm{H}-\mathrm{NMR}\left(400 \mathrm{MHz}\right.$, DMSO- $\left.d_{6}\right) \delta 12.62$ (brs, $\left.1 \mathrm{H}\right), 11.00$ (brs, $\left.1 \mathrm{H}\right), 10.56$ (brs, $\left.1 \mathrm{H}\right)$, 8.20-8.08 (m, 2H), $8.01(\mathrm{~d}, J=8.8 \mathrm{~Hz}, 1 \mathrm{H}), 7.95-7.84(\mathrm{~m}, 3 \mathrm{H}), 7.68-7.63(\mathrm{~m}, 2 \mathrm{H}) .{ }^{13} \mathrm{C}-\mathrm{NMR}(151 \mathrm{MHz}$, DMSO) $\delta 187.76,180.50,150.44,143.33,138.62,136.55,134.99,134.20,133.24,132.79,132.25,132.12$, $131.49,130.24,127.85,126.75,126.38,123.63,113.52,113.36$. MS (ESI) $(m / z): 461.8(\mathrm{M}-\mathrm{H})^{-}$. HRMS (ESI) calcd. for $\mathrm{C}_{20} \mathrm{H}_{11} \mathrm{Cl}_{2} \mathrm{NO}_{6} \mathrm{~S}[\mathrm{M}-\mathrm{H}]^{-}$: 461.9611; found: 461.9614. Purity $99.2 \%$ by HPLC. 
2,6-Dichloro-N-(3,4-dihydroxy-9,10-dioxo-9,10-dihydroanthracen-2-yl) benzenesulfonamide (9k). Red solid, $12 \%$ yield. ${ }^{1} \mathrm{H}-\mathrm{NMR}\left(400 \mathrm{MHz}\right.$, DMSO- $\left.d_{6}\right) \delta 12.62$ (brs, $\left.1 \mathrm{H}\right), 11.00$ (brs, $\left.1 \mathrm{H}\right), 10.56$ (brs, $1 \mathrm{H}$ ), 8.20-8.09 (m, 2H), 7.94-7.83 (m, 2H), 7.72-7.52 (m, 4H). ${ }^{13} \mathrm{C}-\mathrm{NMR}(151 \mathrm{MHz}, \mathrm{DMSO}) \delta 187.71,180.43$, 150.40, 142.57, 134.95, 134.80, 134.29, 134.17, 134.13 (2C), 133.22, 132.78, 131.85(2C), 130.41, 126.75, 126.36, 123.79, 113.20, 112.18. MS (ESI) $(m / z): 461.8(\mathrm{M}-\mathrm{H})^{-}$. HRMS (ESI) calcd. for $\mathrm{C}_{20} \mathrm{H}_{11} \mathrm{Cl}_{2} \mathrm{NO}_{6} \mathrm{~S}$ $[\mathrm{M}-\mathrm{H}]^{-}:$461.9611; found: 461.9601. Purity $95.7 \%$ by HPLC.

2,4,6-Trichloro-N-(3,4-dihydroxy-9,10-dioxo-9,10-dihydroanthracen-2-yl) benzenesulfonamide (91). Yellow solid, $10 \%$ yield. ${ }^{1} \mathrm{H}-\mathrm{NMR}\left(400 \mathrm{MHz}\right.$, DMSO- $\left.d_{6}\right) \delta 12.62$ (brs, $\left.1 \mathrm{H}\right), 10.96$ (brs, $1 \mathrm{H}$ ), 10.73 (brs, 1H), 8.21-8.11 (m, 2H), 7.96-7.86 (m, 4H), $7.66(\mathrm{~s}, 1 \mathrm{H}) .{ }^{13} \mathrm{C}-\mathrm{NMR}(151 \mathrm{MHz}, \mathrm{DMSO}) \delta 187.77,180.49$, 150.48, 143.28, 137.64, 135.25 (2C), 135.00, 134.43, 134.22, 133.27, 132.83, 131.20 (3C), 126.79, 126.41, 123.78, 113.43, 113.21. MS (ESI) $(\mathrm{m} / \mathrm{z})$ : $495.8(\mathrm{M}-\mathrm{H})^{-}$. HRMS (ESI) calcd. for $\mathrm{C}_{20} \mathrm{H}_{10} \mathrm{Cl}_{3} \mathrm{NO}_{6} \mathrm{~S}$ $[\mathrm{M}-\mathrm{H}]^{-}:$495.9222; found: 495.9220 . Purity $99.4 \%$ by HPLC.

4-Bromo-N-(3,4-dihydroxy-9,10-dioxo-9,10-dihydroanthracen-2-yl) benzenesulfonamide (9m). Yellow solid, 65\% yield. ${ }^{1} \mathrm{H}-\mathrm{NMR}\left(400 \mathrm{MHz}\right.$, DMSO- $\left.d_{6}\right) \delta 12.60$ (brs, $\left.1 \mathrm{H}\right), 10.88$ (brs, $\left.1 \mathrm{H}\right), 10.47$ (brs, $1 \mathrm{H}$ ), 8.22-8.11 (m, 2H), 7.95-7.78 (m, 6H), $7.73(\mathrm{~s}, 1 \mathrm{H}) .{ }^{13} \mathrm{C}-\mathrm{NMR}(151 \mathrm{MHz}, \mathrm{DMSO}) \delta 187.77,180.59,150.33$, $142.88,139.50,135.01,134.23,133.29,132.81,132.44$ (2C), 130.65, 128.64 (2C), 127.08, 126.78, 126.39, 123.73, 113.18, 113.05. MS (ESI) $(\mathrm{m} / \mathrm{z}): 471.8(\mathrm{M}-\mathrm{H})^{-}$. HRMS (ESI) calcd. for $\mathrm{C}_{20} \mathrm{H}_{12} \mathrm{BrNO}_{6} \mathrm{~S}$ $[\mathrm{M}-\mathrm{H}]^{-}:$: 471.9496; found: 471.9495 . Purity $96.0 \%$ by HPLC.

2-Bromo-N-(3,4-dihydroxy-9,10-dioxo-9,10-dihydroanthracen-2-yl) benzenesulfonamide (9n). Yellow solid, $46 \%$ yield. ${ }^{1} \mathrm{H}-\mathrm{NMR}\left(400 \mathrm{MHz}, \mathrm{DMSO}-d_{6}\right) \delta 12.63$ (brs, $\left.1 \mathrm{H}\right), 11.10$ (brs, $\left.1 \mathrm{H}\right), 10.24$ (brs, $1 \mathrm{H}$ ), 8.20-8.05 (m, 3H), 7.93-7.84 (m, 3H), 7.67-7.53 (m, 3H). ${ }^{13} \mathrm{C}-\mathrm{NMR}$ (151 MHz, DMSO) $\delta 187.72,180.55$, 150.37, 142.31, 138.96, 135.62, 134.97, 134.74, 134.21, 133.23, 132.80, 130.93, 130.76, 128.28, 126.75, 126.38, 123.75, 119.31, 113.07, 112.14. MS (ESI) $(m / z): 471.8(\mathrm{M}-\mathrm{H})^{-}$. HRMS (ESI) calcd. for $\mathrm{C}_{20} \mathrm{H}_{12} \mathrm{BrNO}_{6} \mathrm{~S}$ $[\mathrm{M}-\mathrm{H}]^{-}:$: 417.9496; found: 417.9498. Purity $97.5 \%$ by HPLC.

3-Bromo-N-(3,4-dihydroxy-9,10-dioxo-9,10-dihydroanthracen-2-yl) benzenesulfonamide (9o). Yellow solid, 54\% yield. ${ }^{1} \mathrm{H}-\mathrm{NMR}\left(400 \mathrm{MHz}, \mathrm{DMSO}-d_{6}\right) \delta 12.60$ (brs, $\left.1 \mathrm{H}\right), 10.99$ (brs, $\left.1 \mathrm{H}\right), 10.53$ (brs, $1 \mathrm{H}$ ), 8.21-8.11 (m, 2H), $8.10(\mathrm{~s}, 1 \mathrm{H}), 7.94-7.83(\mathrm{~m}, 4 \mathrm{H}), 7.71(\mathrm{~s}, 1 \mathrm{H}), 7.57(\mathrm{t}, J=8.0 \mathrm{~Hz}, 1 \mathrm{H}) .{ }^{13} \mathrm{C}-\mathrm{NMR}(151 \mathrm{MHz}$, DMSO) $\delta 187.75,180.55,150.33,142.90,142.23,136.02,134.98,134.19,133.27,132.79,131.56,130.49$, 129.08, 126.76, 126.37, 125.61, 123.71, 122.16, 113.22, 112.97. MS (ESI) $(m / z): 473.8(\mathrm{M}-\mathrm{H})^{-}$.HRMS (ESI) calcd. for $\mathrm{C}_{20} \mathrm{H}_{12} \mathrm{BrNO}_{6} \mathrm{~S}[\mathrm{M}-\mathrm{H}]^{-}:$417.9496; found: 417.9500. Purity $96.1 \%$ by HPLC.

4-Iodo-N-(3,4-dihydroxy-9,10-dioxo-9,10-dihydroanthracen-2-yl) benzenesulfonamide (9p). Yellow solid, 70\% yield. ${ }^{1} \mathrm{H}-\mathrm{NMR}\left(400 \mathrm{MHz}\right.$, DMSO- $d_{6}$ ) $\delta 12.60$ (brs, $1 \mathrm{H}$ ), 10.87 (brs, $\left.1 \mathrm{H}\right), 10.44$ (brs, $\left.1 \mathrm{H}\right), 8.23-8.10$ $(\mathrm{m}, 2 \mathrm{H}), 7.99(\mathrm{~d}, J=8.0 \mathrm{~Hz}, 2 \mathrm{H}), 7.96-7.85(\mathrm{~m}, 2 \mathrm{H}), 7.73(\mathrm{~s}, 1 \mathrm{H}), 7.64(\mathrm{~d}, J=8.0 \mathrm{~Hz}, 2 \mathrm{H}) .{ }^{13} \mathrm{C}-\mathrm{NMR}$ (151 MHz, DMSO) $\delta 187.75,180.61,150.31,142.73,139.85,138.23$ (2C), 135.00, 134.22, 133.29, 132.82, 130.76, 128.27 (2C), 126.78, 126.39, 123.74, 113.12, 112.85, 101.56. MS (ESI) $(m / z): 519.8(\mathrm{M}-\mathrm{H})^{-}$. HRMS (ESI) calcd. for $\mathrm{C}_{20} \mathrm{H}_{12} \mathrm{INO}_{6} \mathrm{~S}[\mathrm{M}-\mathrm{H}]^{-}: 519.9357$; found: 519.9334 . Purity $99.3 \%$ by HPLC.

2-Iodo-N-(3,4-dihydroxy-9,10-dioxo-9,10-dihydroanthracen-2-yl) benzenesulfonamide (9q). Yellow solid, $48 \%$ yield. ${ }^{1} \mathrm{H}-\mathrm{NMR}\left(400 \mathrm{MHz}, \mathrm{DMSO}-d_{6}\right) \delta 12.64$ (brs, $\left.1 \mathrm{H}\right), 11.18(\mathrm{brs}, 1 \mathrm{H}), 10.02(\mathrm{brs}, 1 \mathrm{H}), 8.19-8.06(\mathrm{~m}$, $4 \mathrm{H}), 7.93-7.85(\mathrm{~m}, 2 \mathrm{H}), 7.65(\mathrm{~s}, 1 \mathrm{H}), 7.61(\mathrm{td}, J=1.2,8.0 \mathrm{~Hz}, 1 \mathrm{H}), 7.33(\mathrm{td}, J=1.6,8.0 \mathrm{~Hz}, 1 \mathrm{H}) .{ }^{13} \mathrm{C}-\mathrm{NMR}$ (151 MHz, DMSO) $\delta 187.72,180.58,150.30,142.77,142.13,141.83,134.97,134.22,134.21,133.24,132.81$, 131.01, 130.16, 128.64, 126.76, 126.38, 123.83, 112.96, 111.59, 93.30. MS (ESI) $(m / z): 519.8(\mathrm{M}-\mathrm{H})^{-}$. HRMS (ESI) calcd. for $\mathrm{C}_{20} \mathrm{H}_{12} \mathrm{INO}_{6} \mathrm{~S}[\mathrm{M}-\mathrm{H}]^{-}: 519.9357$; found: 519.9356 . Purity $96.3 \%$ by HPLC.

3.6. Synthesis of ethyl 2-((4-((4-chlorophenyl) sulfonamido)-1-hydroxy-9,10-dioxo-9,10-dihydroanthracen-2-yl) oxy) acetate (12)

Step 1. Compound $\mathbf{1 0}$ was synthesized according to the same route of compound $\mathbf{7}$ in which compound 5 was replaced by compound $\mathbf{6 c}$. Yellow solid, 73\% yield.

Step 2. Compound $\mathbf{1 1}$ was synthesized according to the same route of compound $\mathbf{8}$ in which compound 7 was replaced by compound 10. Purple solid, $93 \%$ yield. 
Step 3. Compound 12 was synthesized according to the same route of $9 \mathbf{i}$ in which compound $\mathbf{8}$ was replaced by compound 11. Red solid, $6 \%$ yield. ${ }^{1} \mathrm{H}-\mathrm{NMR}\left(400 \mathrm{MHz}, \mathrm{DMSO}-\mathrm{d}_{6}\right) \delta 13.37(\mathrm{~s}, 1 \mathrm{H})$, $12.44(\mathrm{~s}, 1 \mathrm{H}), 8.20(\mathrm{~d}, J=7.2 \mathrm{~Hz}, 2 \mathrm{H}), 8.00-7.83(\mathrm{~m}, 4 \mathrm{H}), 7.64(\mathrm{~d}, J=8.0 \mathrm{~Hz}, 2 \mathrm{H}), 7.32(\mathrm{~s}, 1 \mathrm{H}), 5.09(\mathrm{~s}, 2 \mathrm{H})$, $4.25(\mathrm{q}, J=7.2 \mathrm{~Hz}, 2 \mathrm{H}), 1.27(\mathrm{t}, J=7.2 \mathrm{~Hz}, 3 \mathrm{H}) .{ }^{13} \mathrm{C}-\mathrm{NMR}(151 \mathrm{MHz}, \mathrm{DMSO}) \delta 187.87,184.15,167.48$, 153.41, 150.30, 138.86, 137.31, 135.41, 135.23, 134.74, 133.52, 132.12, 129.90 (2C), 128.71 (2C), 127.06, 126.50, 114.75, 109.81, 107.99, 65.44, 61.24, 14.04. MS (ESI) $(\mathrm{m} / \mathrm{z}): 514.2(\mathrm{M}-\mathrm{H})^{-}$. HRMS (ESI) calcd. for $\mathrm{C}_{24} \mathrm{H}_{18} \mathrm{ClNO}_{8} \mathrm{~S}[\mathrm{M}-\mathrm{H}]^{-}: 514.0369$; found: 514.0364 . Purity $96.0 \%$ by HPLC.

3.7. Synthesis of 2-((4-((4-chlorophenyl) sulfonamido)-1-hydroxy-9,10-dioxo-9,10-dihydroanthracen-2-yl) oxy) acetic acid (13)

Compound 13 was synthesized according to the same route of $\mathbf{6 b}$ in which compound $\mathbf{6 a}$ was replaced by compound 12. Red solid, 95\% yield. ${ }^{1} \mathrm{H}-\mathrm{NMR}\left(400 \mathrm{MHz}, \mathrm{DMSO}-\mathrm{d}_{6}\right) \delta 13.36(\mathrm{~s}, 1 \mathrm{H}), 12.53$ (s, 1H), 8.23-8.13 (m, 2H), 7.98-7.84 (m, 4H), $7.64(\mathrm{~d}, J=8.4 \mathrm{~Hz}, 2 \mathrm{H}), 7.35(\mathrm{~s}, 1 \mathrm{H}), 4.99(\mathrm{~s}, 2 \mathrm{H}) .{ }^{13} \mathrm{C}-\mathrm{NMR}$ (151 MHz, Pyr) $\delta$ 188.75, 185.01, 171.15, 155.76, 152.45, 140.30, 139.12, 137.34, 134.82, 133.21, 130.44 (2C), 129.85 (2C), 127.85, 127.27, 121.12, 119.10, 115.52, 110.23, 108.94, 67.24. MS (ESI) $(\mathrm{m} / \mathrm{z}): 485.8(\mathrm{M}-\mathrm{H})^{-}$. HRMS (ESI) calcd. for $\mathrm{C}_{22} \mathrm{H}_{14} \mathrm{ClNO}_{8} \mathrm{~S}$ [M $\left.-\mathrm{H}\right]^{-}:$486.0056; found: 486.0061 . Purity $98.0 \%$ by HPLC.

3.8. Synthesis of ethyl 2-((3-((4-chlorophenyl) sulfonamido)-1-hydroxy-9,10-dioxo-9,10-dihydroanthracen-2-yl) oxy) acetate (14a)

Compound 14a was synthesized according to the same route of $\mathbf{6 c}$ in which compound $\mathbf{5}$ was replaced by compound 9i. Yellow solid, $24 \%$ yield. ${ }^{1} \mathrm{H}-\mathrm{NMR}\left(400 \mathrm{MHz}, \mathrm{DMSO}-d_{6}\right) \delta 12.74(\mathrm{~s}, 1 \mathrm{H})$, $10.59(\mathrm{~s}, 1 \mathrm{H}), 8.22-8.09(\mathrm{~m}, 2 \mathrm{H}), 8.01-7.87(\mathrm{~m}, 4 \mathrm{H}), 7.83-7.68(\mathrm{~m}, 3 \mathrm{H}), 4.85(\mathrm{~s}, 2 \mathrm{H}), 4.19(\mathrm{q}, J=7.2 \mathrm{~Hz}$, 2H), $1.22(\mathrm{t}, J=7.2 \mathrm{~Hz}, 3 \mathrm{H}) .{ }^{13} \mathrm{C}-\mathrm{NMR}(151 \mathrm{MHz}, \mathrm{DMSO}) \delta 187.53,180.83,169.46,154.03,139.72,138.49$, $138.21,136.81,135.08,134.58,132.87,132.72,129.71(2 \mathrm{C}), 128.70(2 \mathrm{C}), 128.46,126.88,126.45,113.29,109.22$, 68.53, 60.98, 13.98. MS (ESI) $(m / z): 514.0(\mathrm{M}-\mathrm{H})^{-}$. HRMS (ESI) calcd. for $\mathrm{C}_{24} \mathrm{H}_{18} \mathrm{ClNO}_{8} \mathrm{~S}[\mathrm{M}-\mathrm{H}]^{-}$: 514.0369; found: 514.0363 . Purity $95.6 \%$ by HPLC.

3.9. Synthesis of 2-((3-((4-chlorophenyl) sulfonamido)-1-hydroxy-9,10-dioxo-9,10-dihydroanthracen-2-yl) oxy) acetic acid $\mathbf{( 1 4 b )}$

Compound $\mathbf{1 4 b}$ was synthesized according to the same route of $\mathbf{6 b}$ in which compound $\mathbf{6 a}$ was replaced by compound 14a. Yellow solid, 95\% yield. ${ }^{1} \mathrm{H}-\mathrm{NMR}\left(400 \mathrm{MHz}, \mathrm{DMSO}-d_{6}\right) \delta 12.72(\mathrm{~s}, 1 \mathrm{H})$, 8.19-8.10 (m, 2H), 7.97-7.87 (m, 4H), $7.75(\mathrm{~s}, 1 \mathrm{H}), 7.71(\mathrm{~d}, J=8.4 \mathrm{~Hz}, 2 \mathrm{H}), 4.80(\mathrm{~s}, 2 \mathrm{H}) .{ }^{13} \mathrm{C}-\mathrm{NMR}$ (151 MHz, DMSO) $\delta 187.49,180.86,171.92,154.23,139.93,138.53,138.14,137.00,135.06,134.59,132.86$, 132.74, 129.76 (2C), 128.67 (2C), 128.58, 126.89, 126.45, 113.28, 108.86, 68.92. MS (ESI) ( $\mathrm{m} / \mathrm{z}): 486.0$ $(\mathrm{M}-\mathrm{H})^{-}$. HRMS (ESI) calcd. for $\mathrm{C}_{22} \mathrm{H}_{14} \mathrm{ClNO}_{8} \mathrm{~S}[\mathrm{M}-\mathrm{H}]^{-}$: 486.0056; found: 486.0049. Purity $99.0 \%$ by HPLC.

3.10. Synthesis of 4-chloro-N-(4-hydroxy-3-methoxy-9,10-dioxo-9,10-dihydroanthracen-2-yl) benzenesulfonamide (14c)

Compound $9 \mathrm{i}(215 \mathrm{mg}, 0.5 \mathrm{mmol}), \mathrm{K}_{2} \mathrm{CO}_{3}(70 \mathrm{mg}, 0.5 \mathrm{mmol})$ and iodomethane $(70 \mathrm{mg}, 12 \mathrm{mmol})$ were added to DMF (2.5 mL) and mixture was stirred at room temperature. The mixture was added to $10 \%$ aqueous $\mathrm{HCl}(20 \mathrm{~mL})$. The suspension was extracted with ethyl acetate and the organic phase was washed with brine, dried over anhydrous $\mathrm{Na}_{2} \mathrm{SO}_{4}$ and filtered. The residue was purified by silica chromatography after removal of ethyl acetate to afford $14 \mathrm{c}$ as a yellow solid (115 mg, 52\%). ${ }^{1} \mathrm{H}-\mathrm{NMR}$ (400 MHz, DMSO- $\left.d_{6}\right) \delta 12.68$ (brs, $\left.1 \mathrm{H}\right), 11.02$ (brs, $\left.1 \mathrm{H}\right), 8.28-8.14(\mathrm{~m}, 2 \mathrm{H}), 7.99-7.89(\mathrm{~m}, 2 \mathrm{H}), 7.70(\mathrm{q}$, $J=8.5 \mathrm{~Hz}, 4 \mathrm{H}), 7.45(\mathrm{~s}, 1 \mathrm{H}), 3.20(\mathrm{~s}, 3 \mathrm{H}) .{ }^{13} \mathrm{C}-\mathrm{NMR}(151 \mathrm{MHz}, \mathrm{DMSO}) \delta 188.19,180.25,151.50,150.13$, 138.19, 137.11, 135.30, 134.32, 133.37, 132.82, 132.10, 129.46 (2C), 129.16 (2C), 126.88, 126.62, 122.74, $121.75,115.43,37.30$. MS (ESI) $(m / z): 442.0(\mathrm{M}-\mathrm{H})^{-}$. HRMS (ESI) calcd. for $\mathrm{C}_{21} \mathrm{H}_{14} \mathrm{ClNO}_{6} \mathrm{~S}[\mathrm{M}-$ $\mathrm{H}]^{-}:$442.0158; found: 442.0162 . Purity $100 \%$ by HPLC. Purity $97.0 \%$ by HPLC. 
3.11. Synthesis of 4-chloro-N-(4-hydroxy-3-methoxy-9,10-dioxo-9,10-dihydroanthracen-2-yl)-N-methylbenzenesulfonamide (14d)

Compound 14d was synthesized according to the same route of $14 \mathrm{c}$ in which room temperature was raised to $40{ }^{\circ} \mathrm{C}$. Orange solid, 50\% yield. ${ }^{1} \mathrm{H}-\mathrm{NMR}\left(400 \mathrm{MHz}, \mathrm{DMSO}-d_{6}\right) \delta 12.79(\mathrm{~s}, 1 \mathrm{H}), 8.28-8.13$ $(\mathrm{m}, 2 \mathrm{H}), 8.00-7.92(\mathrm{~m}, 2 \mathrm{H}), 7.76(\mathrm{q}, \mathrm{J}=8.4 \mathrm{~Hz}, 4 \mathrm{H}), 7.40(\mathrm{~s}, 1 \mathrm{H}), 3.80(\mathrm{~s}, 3 \mathrm{H}), 3.23(\mathrm{~s}, 3 \mathrm{H}) .{ }^{13} \mathrm{C}-\mathrm{NMR}$ (151 MHz, DMSO) $\delta 188.22,180.56,155.48,150.66,138.57,138.37,136.79,135.40,134.67,132.96,132.83$, 129.62 (2C), 129.23 (2C), 127.10, 126.96, 126.72, 119.91, 116.60, 60.65, 38.12. MS (ESI) $(\mathrm{m} / \mathrm{z}): 456.0$ $(\mathrm{M}-\mathrm{H})^{-}$. HRMS (ESI) calcd. for $\mathrm{C}_{22} \mathrm{H}_{16} \mathrm{ClNO}_{6} \mathrm{~S}[\mathrm{M}-\mathrm{H}]^{-}:$456.0314; found: 456.0305. Purity 100\% by HPLC.

\subsection{Synthesis of 4-((4-chlorophenyl) sulfonyl)-12-hydroxy-3,4-dihydro-2H-anthra[2,3-b][1,4]-oxazine-} 6,11-dione (14e)

Compound 14e was synthesized according to the same route of $14 \mathrm{c}$ in which iodomethane was replaced by 1,2-dibromoethane. Orange solid, 32\% yield. ${ }^{1} \mathrm{H}-\mathrm{NMR}\left(400 \mathrm{MHz}, \mathrm{DMSO}-d_{6}\right) \delta 12.63(\mathrm{~s}$, $1 \mathrm{H}), 8.18(\mathrm{t}, J=8.2 \mathrm{~Hz}, 2 \mathrm{H}), 8.11(\mathrm{~s}, 1 \mathrm{H}), 7.98-7.88(\mathrm{~m}, 2 \mathrm{H}), 7.84(\mathrm{~d}, J=8.4 \mathrm{~Hz}, 2 \mathrm{H}), 7.72(\mathrm{~d}, J=8.4 \mathrm{~Hz}$, $2 \mathrm{H}), 4.06(\mathrm{dd}, J=4.4,16.8 \mathrm{~Hz}, 4 \mathrm{H}) .{ }^{13} \mathrm{C}-\mathrm{NMR}(151 \mathrm{MHz}, \mathrm{DMSO}) \delta 187.52,180.70,151.78,140.17,139.33$, 136.21, 135.07, 134.43, 133.27, 132.79, 130.21(2C), 129.30, 129.04(2C), 126.92, 126.55, 124.47, 113.41, 112.82, 64.03, 43.77. MS (ESI) $(m / z): 456.0(\mathrm{M}+\mathrm{H})^{+}$. HRMS (ESI) calcd. for $\mathrm{C}_{22} \mathrm{H}_{14} \mathrm{ClNO}_{6} \mathrm{~S}[\mathrm{M}+\mathrm{H}]^{+}: 456.0303$; found: 456.0307 . Purity $100 \%$ by HPLC.

3.13. Synthesis of 4-chloro-N-(3-(2-hydrazinyl-2-oxoethoxy)-4-hydroxy-9,10-dioxo-9,10-dihydroanthracen-2-yl) benzenesulfonamide (14f)

Compound 14a (20 mg, $0.04 \mathrm{mmol})$ and hydrazine hydrate $(85 \%, 5 \mu \mathrm{L}, 0.16 \mathrm{mmol})$ were added to ethanol $(1.5 \mathrm{~mL})$ and mixture was stirred at $76{ }^{\circ} \mathrm{C}$. A yellow solid $(14 \mathrm{mg}, 72 \%)$ of $\mathbf{1 4 f}$ was obtained by filtration. ${ }^{1} \mathrm{H}-\mathrm{NMR}\left(400 \mathrm{MHz}, \mathrm{DMSO}-d_{6}\right) \delta 12.95$ (brs, $\left.1 \mathrm{H}\right), 8.43-7.36(\mathrm{~m}, 9 \mathrm{H}), 4.53(\mathrm{~s}, 2 \mathrm{H}) .{ }^{13} \mathrm{C}-\mathrm{NMR}$ (151 MHz, DMSO) $\delta 187.43,180.96,168.15,154.79,140.49,138.43,135.10,134.98,134.67,132.89,132.81$, 129.78 (2C), 129.08, 128.64 (2C), 128.51, 126.95, 126.49, 113.41, 109.56, 70.47. MS (ESI) $(\mathrm{m} / \mathrm{z}): 500.0$ $(\mathrm{M}-\mathrm{H})^{-}$. HRMS (ESI) calcd. for $\mathrm{C}_{22} \mathrm{H}_{16} \mathrm{ClN}_{3} \mathrm{O}_{7} \mathrm{~S}[\mathrm{M}-\mathrm{H}]^{-}:$500.0325; found: 500.0340. Purity 95.1\% by HPLC.

\subsection{Synthesis of 3-((4-chlorophenyl) sulfonamido)-1-hydroxy-9,10-dioxo-9,10-dihydroanthracen-2-yl methanesulfonate $\mathbf{( 1 4 g )}$}

Compound $9 \mathrm{i}(107 \mathrm{mg}, 0.25 \mathrm{mmol})$ and DIPEA (50 $\mu \mathrm{L}, 0.3 \mathrm{mmol})$ were added to pyridine $(1.5 \mathrm{~mL})$, and then methanesulfonyl chloride $(23 \mu \mathrm{L}, 0.3 \mathrm{mmol})$ was added to the mixture. After being stirred at room temperature for $4 \mathrm{~h}$, the mixture was added to $10 \%$ aqueous $\mathrm{HCl}(20 \mathrm{~mL})$. The suspension was extracted with ethyl acetate and the organic phase was washed with brine, dried over anhydrous $\mathrm{Na}_{2} \mathrm{SO}_{4}$ and filtered. The residue was purified by silica chromatography after removal of ethyl acetate to afford $14 \mathrm{~g}$ as a yellow solid $(83 \mathrm{mg}, 65 \%)$. ${ }^{1} \mathrm{H}-\mathrm{NMR}\left(400 \mathrm{MHz}, \mathrm{DMSO}-d_{6}\right) \delta 12.81(\mathrm{~s}, 1 \mathrm{H}), 8.23-8.11$ $(\mathrm{m}, 2 \mathrm{H}), 8.00-7.90(\mathrm{~m}, 4 \mathrm{H}), 7.72(\mathrm{~d}, J=8.4 \mathrm{~Hz}, 2 \mathrm{H}), 7.64(\mathrm{~s}, 1 \mathrm{H}), 3.61(\mathrm{~s}, 3 \mathrm{H}) .{ }^{13} \mathrm{C}-\mathrm{NMR}(151 \mathrm{MHz}$, DMSO) $\delta 186.86,180.93,155.49,138.86,138.32,135.07,134.76,132.83$ (2C), 131.81, 131.31, 129.70 (3C), 128.79, (3C) 126.98, 126.50, 109.55, 40.45. MS (ESI) $(m / z): 505.8(\mathrm{M}-\mathrm{H})^{-}$. HRMS (ESI) calcd. for $\mathrm{C}_{21} \mathrm{H}_{14} \mathrm{ClNO}_{8} \mathrm{~S}_{2}[\mathrm{M}-\mathrm{H}]^{-}:$505.9777; found: 505.9791. Purity $99.5 \%$ by HPLC.

\subsection{PGAM1 Enzymatic Assay}

Firstly, $1 \mu \mathrm{L}$ inhibitor in dimethyl sulfoxide (DMSO) incubated with $49 \mu \mathrm{L} 4.6 \mathrm{nM}$ recombinant PGAM1, then $50 \mu \mathrm{L}$ enzyme mix containing 3 units/mL enolase (Sigma-Aldrich, Saint Louis, MO, USA), 3 units/mL recombinant pyruvate kinase M2 (Sigma-Aldrich), 0.6 units/mL recombinant lactatdehydrogenase (LDH, Sigma-Aldrich), $100 \mathrm{mM}$ Tris- $\mathrm{HCl}, 100 \mathrm{mM} \mathrm{KCl}, 5 \mathrm{mM} \mathrm{MgCl} 2,1 \mathrm{mM}$ adenosine diphosphate (ADP), $0.2 \mathrm{mM}$ the reduced form of nicotinamide adenine dinucleotide (NADH) and $4 \mathrm{mM}$ 3PG was added. PGAM1 activity was measured as the decrease in OD at $340 \mathrm{~nm}$. In addition, 
we performed a counter screening in which $4 \mathrm{mM} 2 \mathrm{PG}$ was added to $50 \mu \mathrm{L}$ reaction mix containing the indicated inhibitor, 3 units $/ \mathrm{mL}$ enolase (Sigma-Aldrich), 3 units $/ \mathrm{mL}$ recombinant pyruvate kinase M2 (Sigma-Aldrich), 0.6 units $/ \mathrm{mL}$ recombinant LDH (Sigma-Aldrich), $100 \mathrm{mM}$ Tris-HCl, $100 \mathrm{mM} \mathrm{KCl}$, $5 \mathrm{mM} \mathrm{MgCl}_{2}, 1 \mathrm{mM}$ ADP and $0.2 \mathrm{mM} \mathrm{NADH}$. The other three enzyme activity was measured as the decrease in OD at $340 \mathrm{~nm}$ [17].

\subsection{Constructions of Mutations of PGAM1}

The wild-type plasmid was a gift from Department of Chemistry and Institute for Biophysical Dynamics, University of Chicago (Chicago, IL, USA). To obtain PGAM1 F22A/R116H/R191H mutant construct, the quick change kit (\#KOD-401, TOYOBO, Osaka, Japan) was used to make site-specific mutagenesis, which confirmed by standard DNA sequencing methods. The primers used for the mutation were listed below:

PGAM1 F22A Forward: GAACCTGGAGAACCGCGCCAGCGGCTGGTACGAC PGAM1 F22A Reverse: GTCGTACCAGCCGCTGGCGCGGTTCTCCAGGTTC PGAM1 K116H Forward: CAGGTGAAGATCTGGCACCGCTCCTATGATGTCC PGAM1 K116H Reverse: GGACATCATAGGAGCGGTGCCAGATCTTCATCTG PGAM1 K119H Forward: CATGGCAACAGCCTCCACGGCATTGTCAAGCAT PGAM1 K119H Reverse: ATGCTTGACAATGCCGTGGAGGCTGTTGCCATG

\subsection{Protein Purification, Crystallization, Data Collection and Structure Determination}

The C-terminal His 6 -tagged PGAM1 was expressed and affinity purified following the reported protocols [22]. The crystals of PGAM1 were obtained using the hanging drop vapor-diffusion method at $16{ }^{\circ} \mathrm{C}$ in a crystallization buffer containing of $8 \%(w / v)$ PEG3350 and $100 \mathrm{mM}$ MES 6.0. To obtain the co-crystal of PGAM1 with compound $9 \mathbf{i}$, the crystals of PGAM1 were soaked in stock solution containing $500 \mu \mathrm{M}$ 9i for $2 \mathrm{~h}$. Crystals were then cryo-protected by brief soaking in a mixture solution of crystallization buffer:glycerol (76:24) and quickly cooled in liquid nitrogen. Diffraction data were collected at beamline BL17U1, BL18U1 and BL19U1 in the Shanghai Synchrotron Radiation Facility (SSRF, Shanghai, China) using an X-ray beam of wavelength $0.97851 \AA$ A. The data was handled with HKL3000 [41] and the structure was determined by using molecular replacement in CCP4 [42] via an initial model of PGAM1 derived from PDB entry 4GPZ [22]. The model was then refined to $1.98 \AA$ resolution using Phenix. The ligand restraints were generated using eLBOW in Phenix and manual rebuilding of the model was completed using the molecular graphics program COOT [43] according to the electronic density. All the graphs were plotted by Pymol (DeLano Scientific LLC, San Carlos, CA, USA).

\subsection{Cell Viability Assays}

The H1299 cell line was obtained from Guangzhou Jenniobio Biotechnology Co., Ltd. (Guangzhou, China), PC9 and A549 cells were gifted from Deng Jiong's lab in the School of Medicine, Shanghai Jiao Tong University, Shanghai, China). H1299, PC9 cells were cultured in RPMI-1640 medium and A549 cells were cultured in F-12K medium containing 10\% fetal bovine serum (FBS), 100 units $/ \mathrm{mL}$ of Penicillin and $100 \mu \mathrm{g} / \mathrm{mL}$ Streptomycin. In cell viability assays, $2000 \mathrm{H} 1299$ cells, A549 cells or 1000 PC9 cells per well were seeded in 96-well plate. After attachment for $24 \mathrm{~h}$, the cells were treated with indicated inhibitor for $72 \mathrm{~h}$. After incubation with $0.5 \mathrm{mg} / \mathrm{mL}$ methylthiazolyldiphenyl-tetrazolium bromide (MTT) for $4 \mathrm{~h}$, cell viability was measured as the OD at $570 \mathrm{~nm}$.

\section{Conclusions}

In summary, we designed and synthesized 31 anthraquinone derivatives as PGAM1 inhibitors. The SAR study focused on the effect of 1,2-substituents, 3-substituents, 2,4-substituents, and 2,3-substituents on the anthraquinone core scaffold activity against PGAM1. Among them, 3- 
sulfonamide substituents but not 4-substituents on the anthraquinone scaffold were essential for maintaining potency, which was further revealed by the X-ray structure of PGAM1 complexed with compound $9 \mathbf{i}$ with $\mathrm{IC}_{50}$ value of $0.27 \mu \mathrm{M}$. In addition, modifications of the hydroxyl of alizarin disrupted the $\pi$-cation interaction of PGAM1 and the anthraquinone derivatives which led to the loss of potency. Taken as a whole, the SAR study of anthraquinone derivatives against PGAM1 provided useful information for further discovery of PGAM1 inhibitors.

Supplementary Materials: The following are available online, Figure S1: The activity of PGAM1 mutant.

Author Contributions: conceptualization, K.H., D.Y. and L.Z.; chemistry, K.H. and H.L.; biological assay, K.H. and L.J.; structure determination, L.J. and L.Z.; writing—original draft preparation, K.H.; writing-review and editing, K.H., D.Y. and L.Z.

Funding: This research was funded by the Chinese National Natural Science Foundation (grant no. 21472026, 21877014), the Shanghai Municipal Committee of Science and Technology(14XD1400300), the program for Shanghai Rising Star (15QA1400300), and the open grant of the State Key Laboratory of Bio-organic and Natural Products Chemistry, CAS.

Acknowledgments: We thank Deng Jiong's lab in the School of Medicine, Shanghai Jiao Tong University for providing the A549 and PC9 cells.

Conflicts of Interest: The authors declare no conflict of interest.

\section{References}

1. Warburg, O.; Posener, K.; Negelein, E. Ueber den stoffwechsel der tumoren. Biochem. Z. 1924, 152, 319-344.

2. Warburg, O. On the origin of cancer cells. Science 1956, 123, 309-314. [CrossRef] [PubMed]

3. Hanahan, D.; Weinberg, R.A. Hallmarks of cancer: The next generation. Cell 2011, 144, 646-674. [CrossRef] [PubMed]

4. Vander Heiden, M.G.; DeBerardinis, R.J. Understanding the Intersections between Metabolism and Cancer Biology. Cell 2017, 168, 657-669. [CrossRef] [PubMed]

5. DeNicola, G.M.; Cantley, L.C. Cancer's fuel choice: New flavors for a picky eater. Mol. Cell 2015, 60, 514-523. [CrossRef] [PubMed]

6. Pavlova, N.N.; Thompson, C.B. The Emerging Hallmarks of Cancer Metabolism. Cell Metab. 2016, 23, 27-47. [CrossRef] [PubMed]

7. Schulze, A.; Harris, A.L. How cancer metabolism is tuned for proliferation and vulnerable to disruption. Nature 2012, 491, 364-373. [CrossRef] [PubMed]

8. Dang, C.V. Links between metabolism and cancer. Genes Dev. 2012, 26, 877-890. [CrossRef] [PubMed]

9. Kroemer, G.; Pouyssegur, J. Tumor cell metabolism: Cancer's Achilles' heel. Cancer Cell 2008, 13, 472-482. [CrossRef] [PubMed]

10. DeBerardinis, R.J.; Lum, J.J.; Hatzivassiliou, G.; Thompson, C.B. The biology of cancer: Metabolic reprogramming fuels cell growth and proliferation. Cell Metab. 2008, 7, 11-20. [CrossRef] [PubMed]

11. Vander Heiden, M.G.; Cantley, L.C.; Thompson, C.B. Understanding the Warburg effect: The metabolic requirements of cell proliferation. Science 2009, 324, 1029-1033. [CrossRef] [PubMed]

12. Cantor, J.R.; Sabatini, D.M. Cancer cell metabolism: One hallmark, many faces. Cancer Discov. 2012, 2, 881-898. [CrossRef] [PubMed]

13. Hay, N. Reprogramming glucose metabolism in cancer: Can it be exploited for cancer therapy? Nat. Rev. Cancer 2016, 16, 635-649. [CrossRef] [PubMed]

14. Vander Heiden, M.G. Targeting cancer metabolism: A therapeutic window opens. Nat. Rev. Drug Discov. 2011, 10, 671-684. [CrossRef] [PubMed]

15. Wolpaw, A.J.; Dang, C.V. Exploiting Metabolic Vulnerabilities of Cancer with Precision and Accuracy. Trends Cell Biol. 2018, 28, 201-212. [CrossRef] [PubMed]

16. Fothergill-Gilmore, L.A.; Watson, H.C. The phosphoglycerate mutases. Adv. Enzymol. Relat. Areas Mol. Biol. 1989, 62, 227-313. [PubMed]

17. Hitosugi, T.; Zhou, L.; Elf, S.; Fan, J.; Kang, H.B.; Seo, J.H.; Shan, C.; Dai, Q.; Zhang, L.; Xie, J.; et al. Phosphoglycerate mutase 1 coordinates glycolysis and biosynthesis to promote tumor growth. Cancer Cell 2012, 22, 585-600. [CrossRef] [PubMed] 
18. Chaneton, B.; Gottlieb, E. PGAMgnam style: A glycolytic switch controls biosynthesis. Cancer Cell 2012, 22, 565-566. [CrossRef] [PubMed]

19. Li, C.; Shu, F.; Lei, B.; Lv, D.; Zhang, S.; Mao, X. Expression of PGAM1 in renal clear cell carcinoma and its clinical significance. Int. J. Clin. Exp. Pathol. 2015, 8, 9410-9415. [PubMed]

20. Peng, X.C.; Gong, F.M.; Chen, Y.; Qiu, M.; Cheng, K.; Tang, J.; Ge, J.; Chen, N.; Zeng, H.; Liu, J.Y. Proteomics identification of PGAM1 as a potential therapeutic target for urothelial bladder cancer. J. Proteomics 2016, 132, 85-92. [CrossRef] [PubMed]

21. Liu, X.; Tan, X.; Liu, P.; Wu, Y.; Qian, S.; Zhang, X. Phosphoglycerate mutase 1 (PGAM1) promotes pancreatic ductal adenocarcinoma (PDAC) metastasis by acting as a novel downstream target of the PI3K/Akt/mTOR pathway. Oncol. Res. 2018, 26, 1123-1131. [CrossRef] [PubMed]

22. Hitosugi, T.; Zhou, L.; Fan, J.; Elf, S.; Zhang, L.; Xie, J.; Wang, Y.; Gu, T.L.; Alečković, M.; LeRoy, G.; et al. Tyr26 phosphorylation of PGAM1 provides a metabolic advantage to tumours by stabilizing the active conformation. Nat. Commun. 2013, 4. [CrossRef] [PubMed]

23. Xu, Y.; Li, F.; Lv, L.; Li, T.; Zhou, X.; Deng, C.X.; Guan, K.L.; Lei, Q.Y.; Xiong, Y. Oxidative stress activates SIRT2 to deacetylate and stimulate phosphoglycerate mutase. Cancer Res. 2014, 74, 3630-3642. [CrossRef] [PubMed]

24. Qu, J.; Sun, W.; Zhong, J.; Lv, H.; Zhu, M.; Xu, J.; Jin, N.; Xie, Z.; Tan, M.; Lin, S.H.; et al. Phosphoglycerate mutase 1 regulates dNTP pool and promotes homologous recombination repair in cancer cells. J. Cell Biol. 2017, 216, 409-424. [CrossRef] [PubMed]

25. Zhang, D.; Jin, N.; Sun, W.; Li, X.; Liu, B.; Xie, Z.; Qu, J.; Xu, J.; Yang, X.; Su, Y.; et al. Phosphoglycerate mutase1 promotes cancer cell migration independent of its metabolic activity. Oncogene 2017, 36, 2900-2909. [CrossRef] [PubMed]

26. Evans, M.J.; Saghatelian, A.; Sorensen, E.J.; Cravatt, B.F. Target discovery in small- molecule cell-based screens by in situ proteome reactivity profiling. Nat. Biotechnol. 2005, 23, 1303-1307. [CrossRef] [PubMed]

27. Evans, M.J.; Morris, G.M.; Wu, J.; Olson, A.J.; Sorensen, E.J.; Cravatt, B.F. Mechanistic and structural requirements for active site labeling of phosphoglycerate mutase by spiroepoxides. Mol. Biosyst. 2007, 3, 495-506. [CrossRef] [PubMed]

28. Li, X.; Tang, S.; Wang, Q.Q.; Leung, E.L.; Jin, H.; Huang, Y.; Liu, J.; Geng, M.; Huang, M.; Yuan, S.; et al. Identification of epigallocatechin-3-gallate as an inhibitor of phosphoglycerate mutase 1. Front. Pharmacol. 2017, 8. [CrossRef] [PubMed]

29. Berletch, J.B.; Liu, C.; Love, W.K.; Andrews, L.G.; Katiyar, S.K.; Tollefsbol, T.O. Epigenetic and genetic mechanisms contribute to telomerase inhibition by EGCG. J. Cell. Biochem. 2008, 103, 509-519. [CrossRef] [PubMed]

30. Gao, Y.; Li, W.; Jia, L.; Li, B.; Chen, Y.C.; Tu, Y. Enhancement of (-)-epigallocatechin-3-gallate and theaflavin-3-3'-digallate induced apoptosis by ascorbic acid in human lung adenocarcinoma SPC-A-1 cells and esophageal carcinoma Eca-109 cells via MAPK pathways. Biochem. Biophys. Res. Commun. 2013, 438, 370-374. [CrossRef] [PubMed]

31. Ma, Y.C.; Li, C.; Gao, F.; Xu, Y.; Jiang, Z.B.; Liu, J.X. Epigallocatechin gallate inhibits the growth of human lung cancer by directly targeting the EGFR signaling pathway. Oncol. Rep. 2014, 31, 1343-1349. [CrossRef] [PubMed]

32. Wang, P.; Jiang, L.; Cao, Y.; Zhang, X.; Chen, B.; Zhang, S.; Huang, K.; Ye, D.; Zhou, L. Xanthone derivatives as phosphoglycerate mutase 1 inhibitors: Design, synthesis, and biological evaluation. Bioorg. Med. Chem. 2018, 26, 1961-1970. [CrossRef] [PubMed]

33. Huang, Q.; Lu, G.; Shen, H.M.; Chung, M.C.; Choon, N.O. Anti-cancer properties of anthraquinones from rhubarb. Med. Res. Rev. 2007, 27, 609-630. [CrossRef] [PubMed]

34. Murdock, K.C.; Child, R.G.; Fabio, P.F.; Angier, R.B.; Wallace, R.E.; Durr, F.E.; Citarella, R.V. Antitumor Agents. I. 1,4-Bis[(aminoalkyl)amino]-9,10-anthracenediones. J. Med. Chem. 1979, 22, 1024-1030. [CrossRef] [PubMed]

35. Shrestha, J.P.; Subedi, Y.P.; Chen, L.; Chang, C.-W.T. A mode of action study of cationic anthraquinone analogs: A new class of highly potent anticancer agents. MedChem Comm 2015, 6, 2012-2022. [CrossRef]

36. Wuthi-udomlert, M.; Kupittayanant, P.; Gritsanapan, W. In vitro evaluation of antifungal activity of anthraquinone derivatives of Senna alata. J. Health Res. 2010, 24, 117-122. 
37. Fosso, M.Y.; Chan, K.Y.; Gregory, R.; Chang, C.-W.T. Library synthesis and antibacterial investigation of cationic anthraquinone analogs. ACS Comb. Sci. 2012, 14, 231-235. [CrossRef] [PubMed]

38. Malik, E.M.; Müller, C.E. Anthraquinones As Pharmacological Tools and Drugs. Med. Res. Rev. 2016, 36, 705-748. [CrossRef] [PubMed]

39. Hunter, C.A.; Low, C.M.R.; Rotger, C.; Vinter, J.G.; Zonta, C. Substituent effects on cation- $\pi$ interactions: A quantitative study. Proc. Natl. Acad. Sci. USA 2002, 99, 4873-4876. [CrossRef] [PubMed]

40. Antonello, C.; Uriarte, E.; Palumbo, M. Diethylaminopropionamido-hydroxy-a nthraquinones as potential anticancer agents: Synthesis and characterization. Arch. Pharm. 1989, 322, 541-544. [CrossRef]

41. Otwinowski, Z.; Minor, W. Processing of X-ray diffraction data collected in oscillation mode. Methods Enzymol. 1997, 276, 307-326.

42. Winn, M.D.; Ballard, C.C.; Cowtan, K.D.; Dodson, E.J.; Emsley, P.; Evans, P.R.; Keegan, R.M.; Krissinel, E.B.; Leslie, A.G.; McCoy, A.; et al. Overview of the CCP4 suite and current developments. Acta Crystallogr. D. Biol. Crystallogr. 2011, 67, 235-242. [CrossRef] [PubMed]

43. Emsley, P.; Cowtan, K. Coot: model-building tools for molecular graphics. Acta Crystallogr. D. Biol. Crystallogr. 2004, 60, 2126-2132. [CrossRef] [PubMed]

Sample Availability: Samples of the compounds are not available from the authors.

(c) 2019 by the authors. Licensee MDPI, Basel, Switzerland. This article is an open access article distributed under the terms and conditions of the Creative Commons Attribution (CC BY) license (http://creativecommons.org/licenses/by/4.0/). 